\title{
Gain-scheduled Wheel Slip Control in Automotive
}

\section{Brake Systems}

\author{
Tor A. Johansen*/**, Idar Petersen*, Jens Kalkkuhl ${ }^{* * *}$ and Jens Lüdemann ${ }^{* * * *}$
}

*SINTEF Electronics and Cybernetics, N-7465 Trondheim, Norway.

** Department of Engineering Cybernetics, Norwegian University of Science and Technology,

N-7491 Trondheim, Norway.

${ }^{* * *}$ DaimlerChrysler, Research and Technology, Stuttgart, Germany.

**** DaimlerChrysler, Research and Technology, Alt-Moabit 96a, D-10559 Berlin, Germany.

\begin{abstract}
A wheel slip controller is developed and experimentally tested in a car equipped with electromechanical brake actuators and a brake-by-wire ABS system. A gain scheduling approach is taken, where the vehicle speed is viewed as a slowly time-varying parameter and the model is linearized about the nominal wheel slip. Gain matrices for the different operating conditions are designed using an LQR approach. The stability and robustness of the controller are demonstrated via Lyapunov theory, frequency analysis and experiments using a test vehicle.
\end{abstract}

\section{INTRODUCTION}

An anti-lock brake system (ABS) controls the slip of each wheel of a vehicle to prevent it from locking such that a high friction is achieved and steerability is maintained. ABS brakes are characterized by robust adaptive behaviour with respect to highly uncertain tyre characteristics and fast changing road surface properties and they have been commercially available in cars for more than 20 years [1], [2].

The introduction of advanced functionalities such as ESP (electronic stability program), drive-by-wire and more sophisticated actuators and sensors offer both new opportunities and requirements for a higher performance in automotive brake systems. The contribution of this work is a study of a model-based design of wheel slip control, extending the preliminary results described in [3]. Here, we consider electromechanical actuators, [4], [5], rather than conventional hydraulic actuators, which allow accurate continuous adjustment of the clamping force.

The wheel slip dynamics are highly nonlinear. Despite this fact, our control design relies on local linearization and gainscheduling. In order to analyze the effects of this simplification, we develop a somewhat idealized Lyapunov based nonlinear stability and robustness analysis, taking into account uncertain tyre friction nonlinearities. In order to also investigate the effects of sampling, communications delays, actuator dynamics and the fundamental limitations in performance, this analysis is comple- 
mented by a classical frequency analysis. Experiments using a test vehicle are included. Other contributions to model-based wheel slip control in ABS can be found in [6], [7], [8], [9], [10], while some discrete/hybrid control approaches for hydraulic actuators are described in [2], [11], [12]. Our work is based on a different control design approach with associated analysis, and is the only one that contains detailed experimental evaluation using a test vehicle.

\section{Modelling}

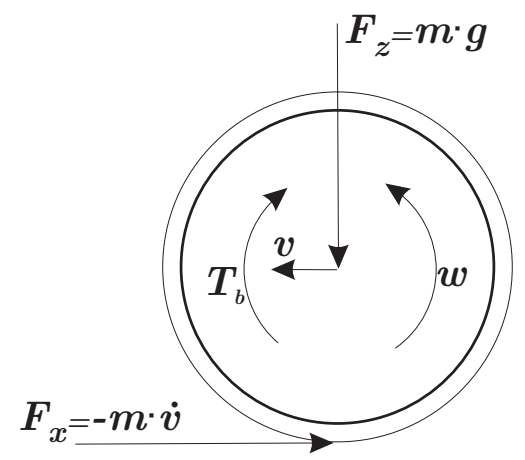

Fig. 1. Quarter car forces and torques.

In this section, we review a mathematical model of the wheel slip dynamics, see also [1], [6], [7]. The problem of wheel slip control is best explained by looking at a quarter car model as shown in Figure 1. The model consists of a single wheel attached to a mass $m$. As the wheel rotates, driven by the inertia of the mass $m$ in the direction of the velocity $v$, a tyre reaction force $F_{x}$ is generated by the friction between the tyre surface and the road surface. The tyre reaction force will generate a torque that results in a rolling motion of the wheel causing an angular velocity $\omega$. A brake torque applied to the wheel will act against the spinning of the wheel causing a negative angular acceleration. The equations of motion of the quarter car are

$$
\begin{aligned}
& m \dot{v}=-F_{x} \\
& J \dot{\omega}=r F_{x}-T_{b} \operatorname{sign}(\omega)
\end{aligned}
$$

where

$v \quad$ longitudinal speed at which the vehicle travels

$\omega \quad$ angular speed of the wheel

$F_{z} \quad$ vertical force

$F_{x} \quad$ tyre friction force

$T_{b} \quad$ brake torque

$r \quad$ wheel radius

$J \quad$ wheel inertia 
The tyre friction force $F_{x}$ is given by

$$
F_{x}=F_{z} \cdot \mu\left(\lambda, \mu_{H}, \alpha\right)
$$

where the friction coefficient $\mu$ is a nonlinear function of

$\lambda \quad$ longitudinal tyre slip

$\mu_{H} \quad$ friction coefficient between tyre and road

$\alpha \quad$ slip angle of the wheel
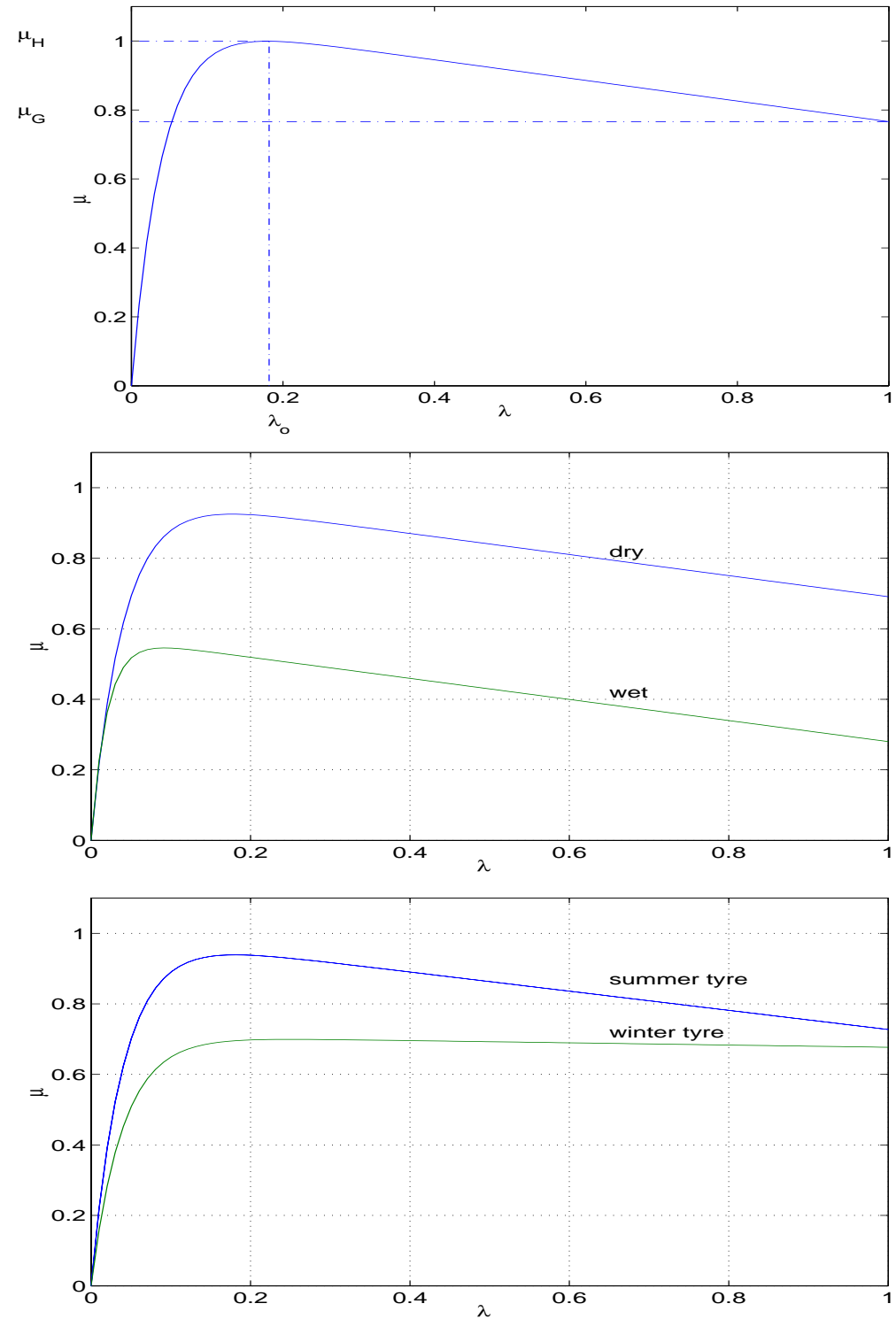

Fig. 2. Typical friction curves.

The longitudinal slip is defined by

$$
\lambda=\frac{v-\omega r}{v}
$$



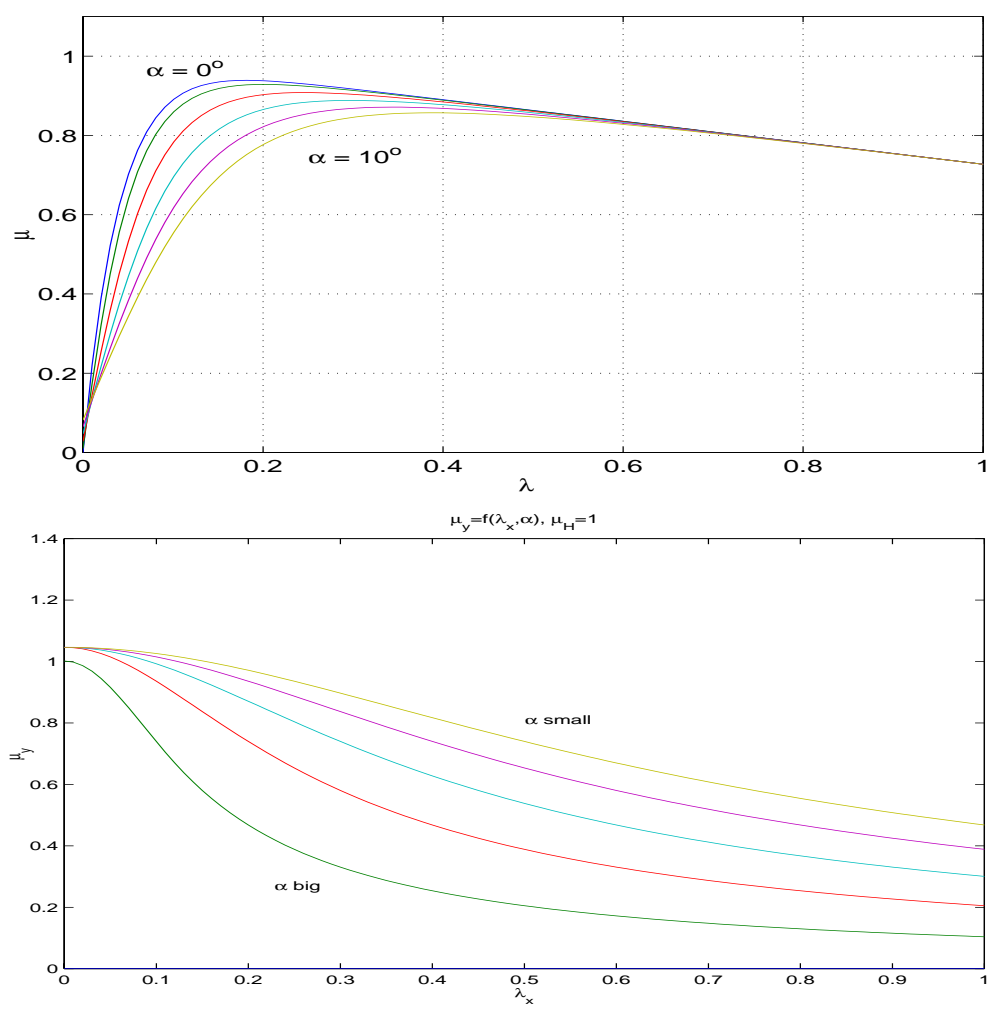

Fig. 3. Tyre side slip/friction curves.

and describes the normalized difference between the vehicle speed $v$ and the speed of the wheel perimeter $\omega r$. The slip value of $\lambda=0$ characterizes the free motion of the wheel where no friction force $F_{x}$ is exerted. If the slip attains the value $\lambda=1$, then the wheel is locked $(\omega=0)$.

The friction coefficient $\mu$ can span over a very wide range, but is generally a differentiable function with the property $\mu(0, \mu H, \alpha)=$ 0 and $\mu\left(\lambda, \mu_{H}, \alpha\right)>0$ for $\lambda>0$. Its typical dependence on slip $\lambda$ is shown in Figure 2 . The upper part shows how the friction coefficient $\mu$ increases with slip $\lambda$ up to a value $\lambda_{0}$, where it attains its maximum value $\mu_{H}$. For higher slip values, the friction coefficient will decrease to a minimum $\mu_{G}$ where the wheel is locked and only the sliding friction will act on the wheel. The dependence of friction on the road condition is shown in the middle part of Figure 2. For wet or icy roads, the maximum friction $\mu_{H}$ is small and the right part of the curve is flatter. The tyre friction curve will also depend on the brand of the tyre, as illustrated in the lower part of Figure 2. In particular, for winter tyres, the curve will cease to have a pronounced peak.

If the motion of the wheel is extended to two dimensions, then the lateral slip of the tyre must also be considered. The slip angle $\alpha$ is the angle between the wheel bearing and the velocity vector of the vehicle. In this case, the longitudinal slip $\lambda_{x}=\frac{v_{x}-\omega r}{v}$ and the lateral slip $\lambda_{y}=\sin \alpha$ are distinguished as well as the corresponding friction coefficients $\mu_{x}$ and $\mu_{y}$. The upper part of Figure 3 shows the dependence of the friction coefficient $\mu_{x}$ on the side slip angle $\alpha$. The lateral force $F_{y}$ depends greatly on the side slip angle $\alpha$ and is shown in the lower part of Figure 3. For large side slip angles, the lateral force gets smaller. This physical phenomenon is the main motivation for ABS brakes, since avoiding high longitudinal slip values will maintain high steerability and 
lateral stability of the vehicle during braking. Achieving this by manual control is difficult because the slip dynamics are fast and open loop unstable when operating at wheel slip values to the right of any peak of the friction curve. We observe that a reasonable tradeoff between high longitudinal friction $\mu_{x}$ and lateral friction $\mu_{y}$ is achieved under all road conditions for longitudinal slip $\lambda_{x}$ close to its peak value on the longitudinal slip curve. Hereafter, for simplification purposes unless otherwise stated, the side slip angle will be considered to be zero with $\mu_{x}=\mu$ and $v_{x}=v$.

Using (1)-(4), for $v>0$ and $\omega \geq 0$ we get the wheel slip dynamics

$$
\begin{gathered}
\dot{\lambda}=-\frac{1}{v}\left(\frac{1}{m}(1-\lambda)+\frac{r^{2}}{J}\right) F_{z} \mu\left(\lambda, \mu_{H}, \alpha\right)+\frac{1}{v} \frac{r}{J} T_{b} \\
\dot{v}=-\frac{1}{m} F_{z} \mu\left(\lambda, \mu_{H}, \alpha\right)
\end{gathered}
$$

Notice that when $v \rightarrow 0$, the dynamics of the open loop system becomes infinitely fast with infinite gain. Hence, the slip controller is switched off for small $v$. The following result shows that the interval $[0,1]$ is a positively invariant set for the wheel slip $\lambda$ under the condition that $v>0$ and $T_{b} \geq 0$ (i.e. there is braking and no traction):

Proposition 1: Consider the system (5)-(6) with $T_{b}(t) \geq 0$ for all $t \geq 0$. If $v(0)>0$ and $\lambda(0) \in[0,1]$, then $\lambda(t) \in[0,1]$ and $\dot{v}(t) \leq 0$ for all $t \geq 0$ where $v(t)>0$

Proof. Note that $\lambda(t)$ is a continuous trajectory since $v(t)>0$. Hence, the possible escape points are $\lambda=0$ and $\lambda=1$. Consider first $\lambda=0$. Since $\mu\left(0, \mu_{H}, \alpha\right)=0$, it follows from (5) that $\dot{\lambda}=\frac{r}{v J} T_{b} \geq 0$ due to $T_{b} \geq 0$. Hence, $\lambda(0) \geq 0$ implies $\lambda(t) \geq 0$ for all $t \geq 0$. Consider next $\lambda=1$. Then, $\omega=0$ and from (2) it follows that $\dot{\omega} \geq 0$ due to the discontinuity sign( $\omega$ ) in (2). From (4), we conclude that $\dot{\lambda}=-r \dot{\omega} / v \leq 0$, which implies $\lambda(t) \leq 1$ for all $t \geq 0$. Finally, note that $\dot{v} \leq 0$ from (1) because $F_{x} \geq 0$ for $\lambda \in[0,1]$

The dynamics of the wheel and car body are given by (5) and (6), respectively. Due to large differences in inertia, the wheel dynamics and car body dynamics will evolve on significantly different time scales. The speed $v$ will change much more slowly than the wheel slip $\lambda$, and $v$ is therefore a natural candidate for gain scheduling. Thus, for the control design, we consider only (5) and regard $v$ as a slow time-varying parameter. A gain scheduled control design requires a set of nominal linearized models for design. Let $\left(\hat{\lambda}, \hat{T}_{b}\right)$ be an equilibrium point for (5) defined by the nominal values $\hat{\alpha}, \hat{F}_{z}$ and $\hat{\mu}_{H}$

$$
\hat{T}_{b}=\left(\frac{J}{m r}(1-\hat{\lambda})+r\right) \hat{F}_{z} \mu\left(\hat{\lambda}, \hat{\mu}_{H}, \hat{\alpha}\right)
$$

The speed-dependent nominal linearized slip dynamics are given by

$$
\dot{\lambda}=\frac{\alpha_{1}}{v}(\lambda-\hat{\lambda})+\frac{\beta_{1}}{v}\left(T_{b}-\hat{T}_{b}\right)+\text { h.o.t. }
$$

where $\alpha_{1}$ and $\beta_{1}$ are linearization constants given by

$$
\begin{aligned}
& \alpha_{1}=-\hat{F}_{z}\left(\frac{1}{m}(1-\hat{\lambda})+\frac{r^{2}}{J}\right) \frac{\partial \mu}{\partial \lambda}\left(\hat{\lambda}, \hat{\mu}_{H}, \hat{\alpha}\right)+\hat{F}_{z} \frac{1}{m} \mu\left(\hat{\lambda}, \hat{\mu}_{H}, \hat{\alpha}\right) \\
& \beta_{1}=\frac{r}{J}
\end{aligned}
$$


Notice that for nominal wheel slip values $\hat{\lambda}$ to the right of any peak of the friction curve we get $\alpha_{1}>0$ such that the open-loop dynamics becomes open-loop unstable. For nominal slip values $\hat{\lambda}$ to the left of any peak, $\alpha_{1}<0$ and the dynamics are open-loop stable. Assuming arbitrary values of $\alpha, F_{z}$ and $\mu_{H}$, the wheel slip dynamics (5) can be written in the form

$$
\dot{x}_{2}=\frac{\phi\left(x_{2}\right)}{v}+\frac{\beta_{1}}{v}\left(T_{b}-T_{b}^{*}\right)
$$

where $x_{2}=\lambda-\lambda^{*}$ and $\lambda^{*}$ is the desired slip (setpoint). Furthermore, we have defined

$$
\phi\left(x_{2}\right)=-\left(\frac{1}{m}\left(1-\lambda^{*}-x_{2}\right)+\frac{r^{2}}{J}\right) F_{z} \mu\left(x_{2}+\lambda^{*}, \mu_{H}, \alpha\right)+\frac{r}{J} T_{b}^{*}
$$

and

$$
T_{b}^{*}=\left(\frac{J}{m r}\left(1-\lambda^{*}\right)+r\right) F_{z} \mu\left(\lambda^{*}, \mu_{H}, \alpha\right)
$$

It can be seen that (10) has an equilibrium point given by $x_{2}=0, T_{b}=T_{b}^{*}$ since $\phi(0)=0$, and the linearized slip model (7) with a perturbation term is written as follows

$$
\dot{x}_{2}=\frac{\alpha_{1}}{v} x_{2}+\frac{\beta_{1}}{v}\left(T_{b}-T_{b}^{*}\right)+\frac{\epsilon_{\mu}\left(x_{2}\right)}{v}
$$

where $\epsilon_{\mu}\left(x_{2}\right)=\phi\left(x_{2}\right)-\alpha_{1} x_{2}$. Eq. (13) will be used later on for control design and analysis.

\section{Control DESIGN AND ANALYSIS}

\section{A. Control problem}

The actual control input is the clamping force $F_{b}$ that is related to the brake torque as $T_{b}=k_{b} F_{b}$, where the constant $k_{b}$ depends on the friction between the brake pads and the brake disc. There are limitations on the clamping force that can be applied to the brake pads by the actuator during braking. The (small) minimum force is to ensure that the brake pads are positioned close to the brake disk with no air-gap. The maximum force is what the actuator is capable of. There is also a rate limit at how fast the torque can be changed by the actuator.

The control problem is to regulate the value of the longitudinal slip $\lambda$ to a given setpoint $\lambda *$ that is either constant or commanded from a higher-level control system. The controller must be robust with respect to uncertainties in the tyre characteristic, brake pads/discs, variations in the road surface conditions, load on the vehicle etc. Integral action or adaptation must be incorporated to remove steady-state error due to model inaccuracies, in particular the unknown road/tyre friction coefficient $\mu_{H}$.

\section{B. Wheel slip control with integral action}

Let the system dynamics (13) be augmented with a slip error integrator $\dot{x}_{1}=\lambda-\lambda^{*}=x_{2}$ such that

$$
\left(\begin{array}{c}
\dot{x}_{1} \\
\dot{x}_{2}
\end{array}\right)=A(v)\left(\begin{array}{c}
x_{1} \\
x_{2}
\end{array}\right)+B(v)\left(u-T_{b}^{*}\right)+W(v) \epsilon_{\mu}\left(x_{2}\right)
$$


where

$$
A(v)=\left(\begin{array}{cc}
0 & 1 \\
0 & \frac{\alpha_{1}}{v}
\end{array}\right), B(v)=\left(\begin{array}{c}
0 \\
\frac{\beta_{1}}{v}
\end{array}\right), W(v)=\left(\begin{array}{c}
0 \\
\frac{1}{v}
\end{array}\right)
$$

The steady-state brake torque $T_{b}^{*}$ depends on road and tyre properties such as $\mu_{H}$ and must therefore be assumed unknown. Hence, we define the control input $u=T_{b}$, and the equilibrium point is

$$
x^{*}=\left(\begin{array}{c}
x_{1}^{*} \\
0
\end{array}\right), u^{*}=T_{b}^{*}
$$

where $x_{1}^{*}$ depends on the controller. This leads to

$$
\dot{x}=A(v)\left(x-x^{*}\right)+B(v)\left(u-u^{*}\right)+W(v) \epsilon_{\mu}\left(x_{2}\right)
$$

Next, define the quadratic cost function for the purpose of local LQ design based on the nominal part of (17):

$$
J(x(t), u[t, \infty))=\int_{t}^{\infty}\left(\left(x(\tau)-x^{*}\right)^{T} Q(v)\left(x(\tau)-x^{*}\right)+\left(u(\tau)-u^{*}\right)^{T} R\left(u(\tau)-u^{*}\right)\right) d \tau
$$

Assuming constant $v$, the optimal control law is given by

$$
\hat{u}=K(v) x
$$

where the gain matrix $K(v)=-R^{-1} B^{T}(v) P(v)$ and we neglect the unknown $x^{*}$ and $u^{*}$ which will be accounted for due to the integral action. The symmetric matrix $P(v)>0$ is defined by the solution to the algebraic Riccati equation for the design:

$$
P(v) A(v)+A^{T}(v) P(v)-2 P(v) B(v) R^{-1} B^{T}(v) P(v)=-Q(v)
$$

The elements of the matrix equation (20) are

$$
\begin{aligned}
\left(\frac{\beta_{1}}{v}\right)^{2} \frac{P_{1,2}^{2}(v)}{R} & =Q_{1,1}(v) \\
P_{1,1}(v)+P_{1,2}(v)\left(\frac{\alpha_{1}}{v}-\left(\frac{\beta_{1}}{v}\right)^{2} \frac{P_{2,2}(v)}{R}\right) & =0 \\
2 P_{1,2}(v)+P_{2,2}(v)\left(\frac{2 \alpha_{1}}{v}-\left(\frac{\beta_{1}}{v}\right)^{2} \frac{P_{2,2}(v)}{R}\right) & =-Q_{2,2}(v)
\end{aligned}
$$

This gives the following solution with $P(v)>0$ :

$$
\begin{aligned}
& P_{1,1}(v)=\frac{\left(\alpha_{1}^{2}+\beta_{1}^{2} R^{-1}\left(Q_{2,2}(v)+\frac{2\left(Q_{1,1}(v) R\right)^{1 / 2}}{\beta_{1}} v\right)\right)^{1 / 2}}{\left(Q_{1,1}(v) R\right)^{-1 / 2} \beta_{1}} \\
& P_{1,2}(v)=\frac{v}{\beta_{1}}\left(Q_{1,1}(v) R\right)^{1 / 2} \\
& P_{2,2}(v)=\frac{\alpha_{1}+\left(\alpha_{1}^{2}+\beta_{1}^{2} R^{-1}\left(Q_{2,2}(v)+\frac{2\left(Q_{1,1}(v) R\right)^{1 / 2}}{\beta_{1}} v\right)\right)^{1 / 2}}{\beta_{1}^{2} R^{-1}} v
\end{aligned}
$$


and the gains

$$
\begin{aligned}
& K_{1}(v)=-\left(Q_{1,1}(v) R^{-1}\right)^{1 / 2} \\
& K_{2}(v)=-\frac{\alpha_{1}+\left(\alpha_{1}^{2}+\beta_{1}^{2} R^{-1}\left(Q_{2,2}(v)+\frac{2\left(Q_{1,1}(v) R\right)^{1 / 2}}{\beta_{1}} v\right)\right)^{1 / 2}}{\beta_{1}}
\end{aligned}
$$

Setting $x_{1}^{*}=T_{b}^{*} / K_{1}(v)$ gives the closed loop dynamics

$$
\dot{\tilde{x}}=(A(v)+B(v) K(v)) \tilde{x}+W(v) \epsilon_{\mu}\left(x_{2}\right)
$$

with $\tilde{x}=x-x^{*}$.

Proposition 2: Consider the system (5) with controller defined by (19). Assume $R>0$ and the smooth matrix-valued function $Q$ satisfies $Q_{1,2}(v)=Q_{2,1}(v)=0, Q_{1,1}(v)>0, Q_{2,2}(v)>0, \frac{d Q_{1,1}(v)}{d v} \geq 0, \frac{d Q_{2,2}(v)}{d v} \geq 0$ for all $v>0$. Moreover, suppose $T_{b}(t) \geq 0$ for all $t \geq 0$ and

$$
\begin{aligned}
D(v) & =P_{1,1}^{\prime}(v) P_{2,2}^{\prime}(v)-P_{1,2}^{\prime}(v) P_{2,1}^{\prime}(v)>0 \\
Q_{1,1}(v)(1-\delta) & >\frac{P_{2,1}(v) C}{v} \\
Q_{2,2}(v) \tilde{x}_{2}^{2}(1-\delta) & >\left(\frac{2}{v} \epsilon_{\mu}\left(\tilde{x}_{2}\right) P_{2,2}(v) \tilde{x}_{2}+\frac{P_{2,1}(v) \epsilon_{\mu}^{2}\left(\tilde{x}_{2}\right)}{v C}\right)
\end{aligned}
$$

are satisfied for all $v>0, \tilde{x}_{2} \in\left[-\lambda^{*}, 1-\lambda^{*}\right]$ and some $C>0$ and $\delta \in(0,1)$. Then, the equilibrium $\tilde{x}=0$ is uniformly exponentially stable.

Proof. Let a Lyapunov function candidate be

$$
V(\tilde{x})=\tilde{x}^{T} P(v) \tilde{x}
$$

Its time-derivative along trajectories of (29)

$$
\dot{V}=\frac{d}{d t} V(\tilde{x}(t))=\tilde{x}^{T}\left(\frac{d P(v)}{d v} \dot{v}\right) \tilde{x}+\dot{\tilde{x}}^{T} P \tilde{x}+\tilde{x}^{T} P \dot{\tilde{x}}
$$

is found by substituting for (17), (19) and (20) in (33):

$$
\dot{V}=\tilde{x}^{T} \frac{d P(v)}{d v} \dot{v} \tilde{x}+\epsilon_{\mu}\left(\tilde{x}_{2}\right)\left(W^{T}(v) P(v) \tilde{x}+\tilde{x}^{T} P(v) W(v)\right)-\tilde{x}^{T} Q(v) \tilde{x}
$$

¿From Proposition 1, it is clear that $\dot{v} \leq 0$. Thus, the negativity of $\tilde{x}^{T}\left(\frac{d P(v)}{d v} \dot{v}\right) \tilde{x}$ requires $P^{\prime}(v)=\frac{d P(v)}{d v}>0$ for all $v>0$. For $P^{\prime}(v)$ to be positive definite, it is sufficient that $P_{1,1}^{\prime}(v)>0$ and $D(v)>0$. Note that since $Q_{1,1}(v), Q_{2,2}(v), \beta_{1}>0$, it follows immediately that $P_{1,1}^{\prime}(v)>0$. Then, (34) becomes:

$$
\begin{aligned}
\dot{V} & \leq \epsilon_{\mu}\left(\tilde{x}_{2}\right)\left(W^{T} P(v) \tilde{x}+\tilde{x}^{T} P(v) W(v)\right)-\tilde{x}^{T} Q(v) \tilde{x} \\
& =-Q_{1,1}(v) \tilde{x}_{1}^{2}-Q_{2,2}(v) \tilde{x}_{2}^{2}+\frac{2}{v} \epsilon_{\mu}\left(\tilde{x}_{2}\right)\left(P_{2,2}(v) \tilde{x}_{2}+P_{2,1}(v) \tilde{x}_{1}\right)
\end{aligned}
$$


To obtain all $\tilde{x}_{1}$-terms in (35) in a quadratic form, we apply Young's inequality $2 a b \leq a^{2} / C+C b^{2}$. Hence,

$$
\dot{V} \leq-Q_{1,1}(v) \tilde{x}_{1}^{2}+\frac{P_{2,1}(v)}{v} \tilde{x}_{1}^{2} C-Q_{2,2}(v) \tilde{x}_{2}^{2}+\frac{2}{v} \epsilon_{\mu}\left(\tilde{x}_{2}\right) P_{2,2}(v) \tilde{x}_{2}+\frac{P_{2,1}(v)}{v} \frac{\epsilon_{\mu}^{2}\left(\tilde{x}_{2}\right)}{C}
$$

Due to (31) and (32) it follows that

$$
\dot{V} \leq-\delta Q_{1,1}(v) \tilde{x}_{1}^{2}-\delta Q_{2,2}(v) \tilde{x}_{2}^{2}
$$

and we conclude that the equilibrium is uniformly exponentially stable [13].

Inequality (31) states that the error weight $Q_{1,1}(v)$ must be sufficiently large, leading to a sufficiently large controller gain. Note that $P_{2,1}(v)$ depends on $Q_{1,1}(v)$, but from (24), it is evident that $P_{2,1}(v)$ increases with $\sqrt{Q_{1,1}(v)}$ such that (31) will indeed hold for a sufficiently large $Q_{1,1}(v)$, except when $v \rightarrow 0$.

Inequality (32) states that the error weight $Q_{2,2}(v)$ must also be sufficiently large, leading to a sufficiently high gain to stabilize the system. From (26), it follows that $P_{2,2}(v)$ increases with $\sqrt{Q_{2,2}(v)}$ such that this is also possible, except for $v \rightarrow 0$.

Notice that $Q_{2,2}(v)\left|\tilde{x}_{2}\right|$ is essentially chosen in (32) to dominate the perturbation $\epsilon_{\mu}\left(\tilde{x}_{2}\right)$. Inequality (32) must be checked with respect to the perturbations $\epsilon_{\mu}$ that are generated by all possible friction curves $\mu(\cdot)$ to ensure robust stability.

Inequality (30) can be seen to be non-restrictive since it is shown in the Appendix that this will always be satisfied for $\alpha_{1}$ close to zero. This corresponds to generating the nominal model by linearizing near the peak of the friction curve. Such a nominal operating point is generally desirable since it means that the friction is maximized, and in section IV-B we will show that high robustness and large stability margins are also achieved this way. Note that the only information on the friction curves utilized in the control design is its slope at the setpoint. If $\alpha_{1}=0$ it is assumed that the setpoint is chosen near any peak of the friction curve.

The constant $C>0$ should be chosen to minimize conservativeness. However, the choice $Q_{1,2}(v)=Q_{2,1}(v)=0$ and taking $P(v)$ from the solution of the algebraic Riccati equation are possibly conservative.

The controller gain $K(v)$ depends on the speed (gain scheduling). ¿From a practical point of view, a useful gain schedule is achieved by letting $\frac{d Q_{1,1}(v)}{d v}>0$ and $\frac{d Q_{2,2}(v)}{d v}>0$, as this reduces the gain as $v \rightarrow 0$. This is necessary to avoid instability due to the unmodelled dynamics since these tend to dominate as $v \rightarrow 0$.

The value of the Lyapunov function $V(\tilde{x}(0), v(0))$ is a measure on the magnitude of the initial transient when the wheel slip controller is switched on when the system is in the state given by $\tilde{x}(0)$ and $v(0)$. Hence, the transient response can be improved by choosing the initial value of the controller state $\tilde{x}_{1}(0)$, such that $V(\tilde{x}(0), v(0))$ is as small as possible. Unfortunately, the information required to minimize the transient requires knowledge of $\mu_{H}$, which is not generally available. However, we shall discuss later how to use estimates of the friction and demonstrate experimentally the benefits, see also [14], [15] for methods describing how to develop systematic initialization and resetting strategies. 

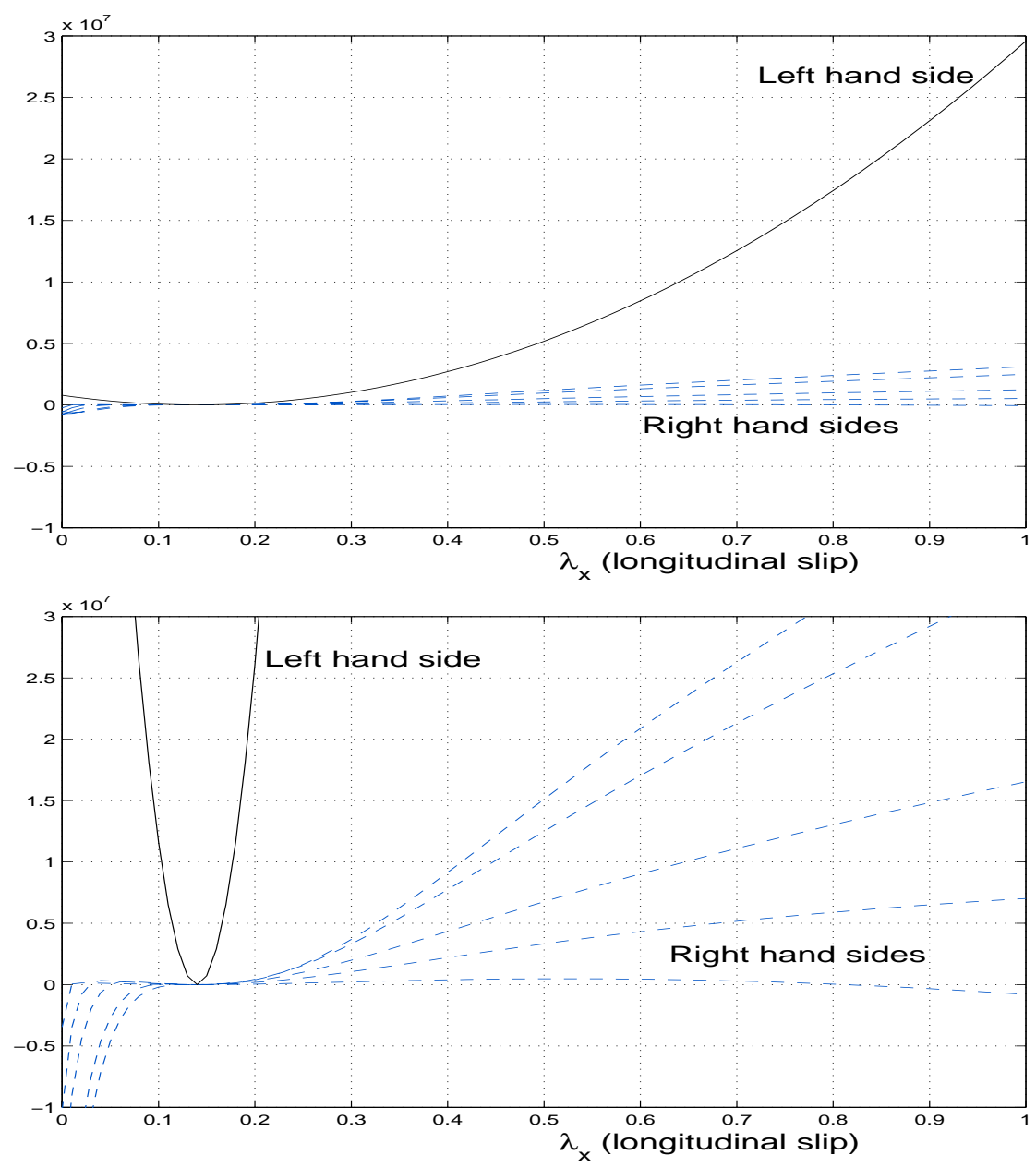

Fig. 4. Illustration of the robust stability requirement, i.e. the left and right hand sides of eq. (32). The top figure is for $v=1 \mathrm{~m} / \mathrm{s}$ and the bottom figure is for $v=32 \mathrm{~m} / \mathrm{s}$.

\section{An idealized design example}

We consider a design example with the following parameters $m=450 \mathrm{~kg}, F_{z}=4414 \mathrm{~N}, \mathrm{r}=0.32 \mathrm{~m}, \mathrm{~J}=1.0 \mathrm{~kg} \mathrm{~m}^{2}$ and the friction model in the upper part of Figure 2. Assuming $\hat{\lambda}=0.14$ and the nominal design $\hat{\mu}_{H}=0.8$ and $\hat{\alpha}=0$, we get $\alpha_{1}=10.2$ and $\beta_{1}=0.32$. We choose $R=1$ and $Q(v)=\tilde{Q} v^{3 / 2}$ with $\tilde{Q}_{1,1}=6 \cdot 10^{9}$ and $\tilde{Q}_{2,2}=40 \cdot 10^{6}$. Note the scaling due to the different magnitudes of $T_{b}$ and $\lambda$. The choice for $Q(v)$ leads to a gain schedule with reduced gain as $v \rightarrow 0$, that is useful to avoid instability due to unmodelled actuator dynamics as $v \rightarrow 0$.

Figure 4 shows that the robust stability requirement (32) is satisfied for all $\tilde{x}_{2} \in\left[-\lambda^{*}, 1-\lambda^{*}\right]$ for a family of typical friction curves. Although curves are shown only for $v=1 \mathrm{~m} / \mathrm{s}$ and $v=32 \mathrm{~m} / \mathrm{s}$, it has been verified that (32) is fulfilled for intermediate values of $v$. The control design also satisfies the stability requirements (31) and (47), and we conclude robust uniform exponential stability of the equilibrium. 


\section{PRACTICAL DESIGN AND IMPLEMENTATION}

\section{A. Test vehicle}

The experimental vehicle is a Mercedes E220 equipped with electromechanical disc brake actuators and a brake-by-wire system, see Figure 5. An early version of this system is described in [4], [5]. The system consists of five electronic control units (ECUs), one for each actuator with individual torque servo controllers, and one central ECU where the four wheel slip controllers run. The brake-by-wire system is based on TTP (time-triggered protocol) communication between sensors, ECUs and actuators. TTP is a synchronous communication protocol with high reliability [4], [16]. The wheel slip controllers are executed with sampling interval of $T_{s}=7 \mathrm{~ms}$. The torque controllers on each wheel runs at $2.33 \mathrm{~ms}$ sampling interval, and the total delay due to synchronous communication is $7 \mathrm{~ms}$ on the sensor side and $7 \mathrm{~ms}$ on the actuator side. In addition, the electromechanical actuator has its internal dynamics, which can be approximated to sufficient accuracy for control design by the following first order discrete-time linear model

$$
T_{b}(t+1)=a_{a c t} T_{b}(t)+b_{a c t} \tilde{T}_{b}(t)
$$

with $a_{a c t}=0.6$ and $b_{a c t}=0.4$, corresponding to an actuator bandwidth of $72 \mathrm{rad} / \mathrm{s} . T_{b}$ is the brake torque, while $\tilde{T}_{b}$ is the brake torque commanded to the actuator servo.

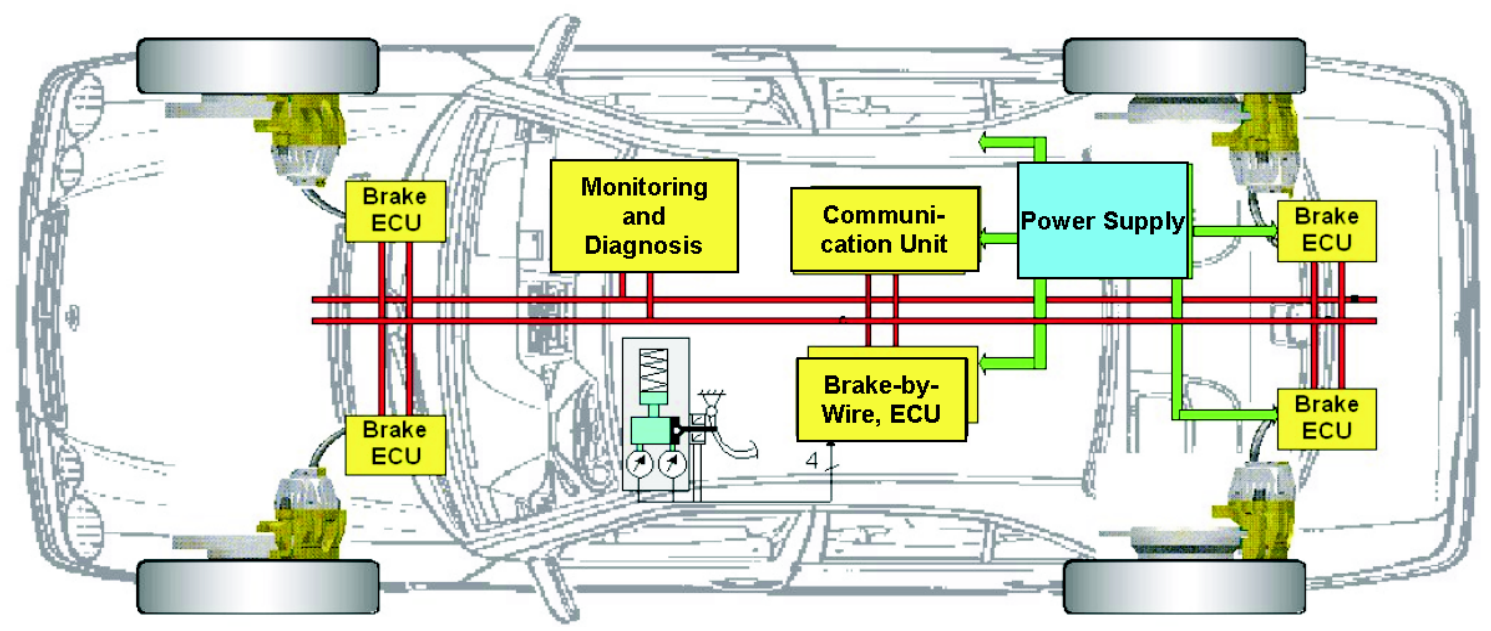

Fig. 5. Diagram of test vehicle with brake-by-wire.

\section{B. Discrete-time design incorporating actuator dynamics}

Both the actuator dynamics and the communications delays introduce fundamental limitations on the achievable performance and the maximum gain that can be tolerated. In order to achieve high performance of the design and take into account the effects of sampling and communication, we discretize and augment the second-order linearized model (14) with an actuator/communication model. We also take the change in commanded clamping torque $u(t)=\tilde{T}_{b}(t)-\tilde{T}_{b}(t-1)$ as the control input, as this simplifies the 
handling of actuator rate constraints in the controller. Moreover, this brings the model in a velocity form that is favourable when implementing a gain scheduled controller [17], [18]. This leads to a 4th order discrete-time linear parameter-varying (LPV) state space model of the form

$$
x(t+1)=\Phi(v) x(t)+\Gamma u(t)
$$

where $x_{1}$ is the integrated slip error, $x_{2}=\lambda-\lambda^{*}$ is the slip error, $x_{3}=T_{b}$ is the brake torque produced by the actuator and $x_{4}=\tilde{T}_{b}$ is the brake torque commanded to the actuator. The speed-dependent model matrices are given by

$$
\Phi(v)=\left(\begin{array}{cccc}
1 & T_{s} & 0 & 0 \\
0 & a_{1}(v) & b_{1}(v) & 0 \\
0 & 0 & a_{a c t} & b_{a c t} \\
0 & 0 & 0 & 1
\end{array}\right), \quad \Gamma=\left(\begin{array}{l}
0 \\
0 \\
0 \\
1
\end{array}\right)
$$

with the following discretization [19]

$$
a_{1}(v)=e^{T_{s} \alpha_{1} / v}, \quad b_{1}(v)=\beta_{1}\left(a_{1}(v)-1\right) / \alpha_{1}
$$

This model is utilized for the design of gain matrices at appropriate operating points. The design method is an explicit constrained LQR that gives an explicit piecewise linear controller that incorporates actuator rate constraints [20]. Thus, if no constraints are active, the controller is of the form

$$
u(t)=K(v) x(t)
$$

The test vehicle has the following nominal parameters: $m=450 \mathrm{~kg}, F_{z}=4414 \mathrm{~N}, \mathrm{r}=0.32 \mathrm{~m}, \mathrm{~J}=1.0 \mathrm{~kg} \mathrm{~m}^{2}$ and the friction model in the upper part of Figure 2. Assuming $\hat{\lambda}=0.14$ and the nominal design $\hat{\mu}_{H}=0.8$ and $\hat{\alpha}=0$, we get $\alpha_{1}=10.2$ and $\beta_{1}=0.32$. We notice that since $\alpha_{1}>0$, this nominal point is on the open-loop unstable part of the friction curve, i.e. to the right of the peak. For the control design, we choose $R=1$ and $Q(v)=\tilde{Q} v^{3 / 2}$ with $\tilde{Q}_{1,1}=8 \cdot 10^{6}$ and all other elements of $\tilde{Q}$ equal to zero. Notice the scaling due to the different magnitudes of $T_{b}$ and $\lambda$. This choice for $Q(v)$ leads to a gain schedule with reduced gain for $K_{1}(v)$ and $K_{2}(v)$ as $v \rightarrow 0$, see Figure 6 . Reduced gain is necessary to avoid instability due to unmodelled dynamics as $v \rightarrow 0$.

For unstable operating points (with setpoint $\lambda^{*}$ to the right of the friction curve peak), it is well known that a minimum gain is necessary to stabilize the open-loop unstable dynamics. Since the communications delay and actuator dynamics impose fundamental limitations on the maximum gain, it is of interest to investigate if all operating points, i.e. all values of $\lambda^{*}$ for all possible friction curves, can be stabilized with acceptable performance. This analysis is carried out most conveniently using a classical frequency analysis based on a linearized model, and a block diagram for the linearized dynamics is shown in Figure 7.

Assume for a moment the setpoint $\lambda^{*}$ corresponds to a slip value near the peak of the friction curve. The family of frequency responses (from slip setpoint to slip, computed based on the block diagram in Figure 7) corresponding to values of $v$ between 0.75 


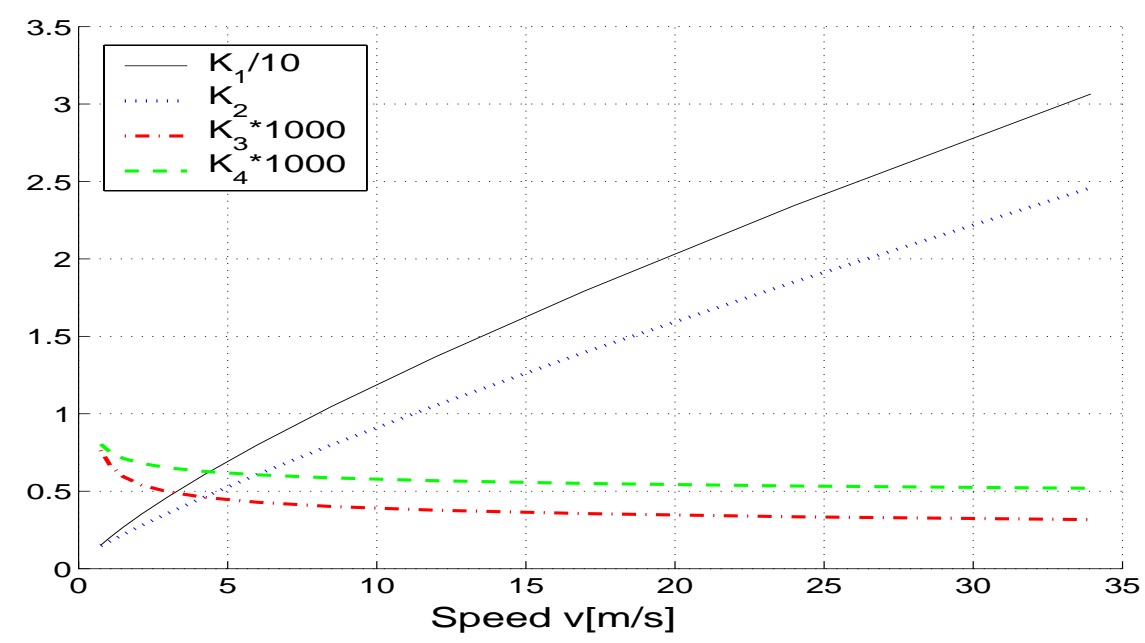

Fig. 6. Gain $K(v)$, as a function of $v$.

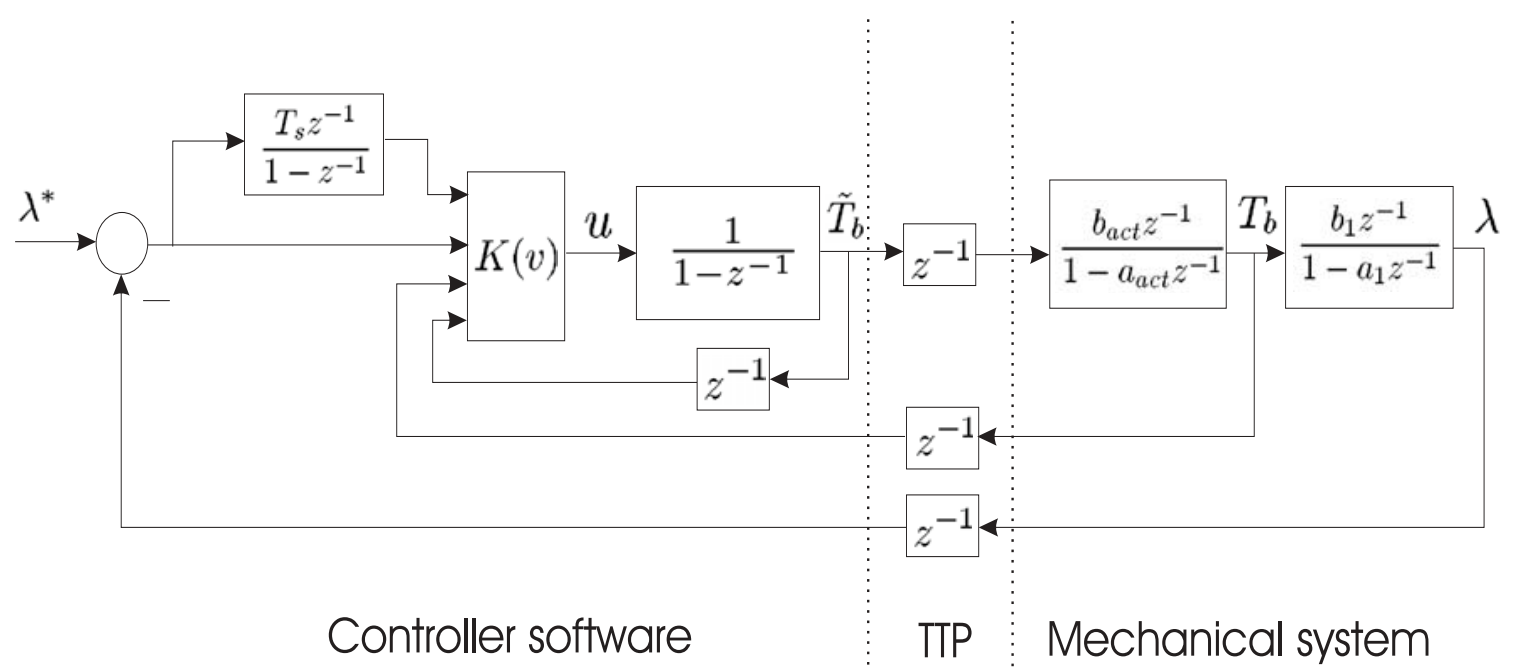

Fig. 7. Block diagram including actuator dynamics and communication delays.

$\mathrm{m} / \mathrm{s}$ and $32 \mathrm{~m} / \mathrm{s}$ are given in Figure 8 . It can be observed that the bandwidth $(-3 \mathrm{~dB}$ frequency) is between $42 \mathrm{rad} / \mathrm{s}$ and $75 \mathrm{rad} / \mathrm{s}$ (depending on $v$ ). If the setpoint is moved sufficiently far to the right of the peak of the friction curve, the closed loop becomes unstable since the gain is not sufficiently large to stabilize the open-loop unstable system. On the other hand, if the setpoint is moved somewhat to the left of the peak of the friction curve, the family of frequency responses changes to Figure 9 . It is observed that the bandwidth decreases significantly, especially for small $v$, indicating a loss of performance. If the setpoint is moved even further left of the peak, a further loss of performance is experienced. Thus, high performance is achieved only if the setpoint is chosen near the peak of the friction curve. At low friction, the peak is less pronounced and the performance and the robustness are not expected to be very sensitive to the choice of setpoint. At high friction, however, the peak is significant and loss of stability will occur if the setpoint is chosen too high ${ }^{1}$, and loss of performance may occur if the setpoint is chosen too low. It must be stressed

\footnotetext{
${ }^{1}$ In experiments we have observed that this situation is characterized by oscillatory behavior with peaks in the wheel slip, similar to how a conventional ABS
} system operates. Hence, the performance and comfort is degraded, but the system still operates in a safe manner. 

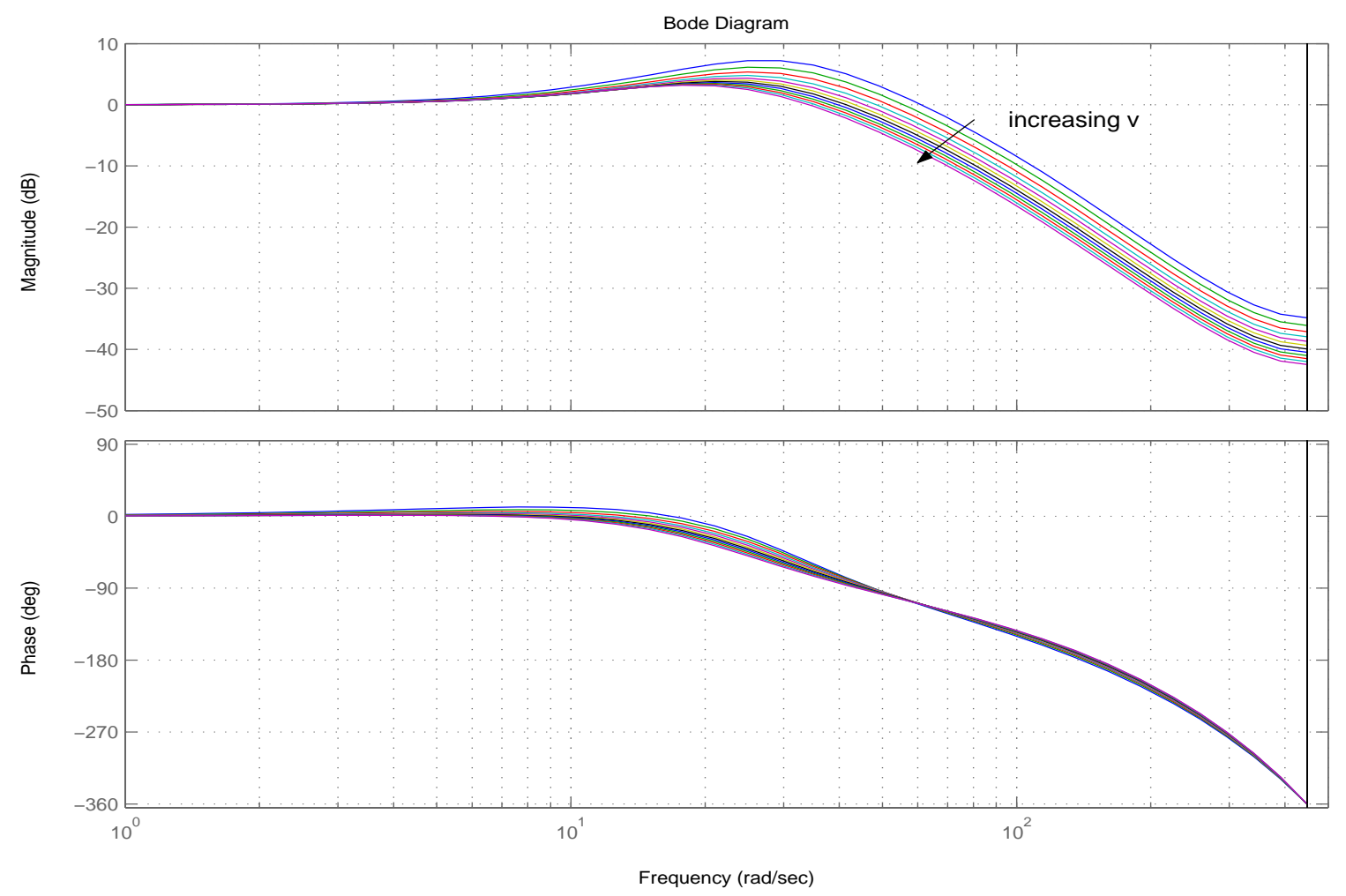

Fig. 8. Transfer function from reference to output, when the setpoint is near the peak of the friction curve.

that this analysis is based on a linearized model and is therefore not valid for transients that are far from the equilibrium. However, it still correctly points out correctly fundamental limitations in performance and robustness, and suggests useful guidelines for selecting the slip setpoint $\lambda^{*}$.

\section{Additional implementation details}

Gain scheduling is implemented by switching gain matrices, where the gain matrices are computed for a finite number of operating points (12 velocities logarithmically spaced between $0.75 \mathrm{~m} / \mathrm{s}$ and $32 \mathrm{~m} / \mathrm{s}$ ). To achieve bumpless transfer, the integrator state $x_{1}$ is reset at the switching instants to achieve a control signal without any discontinuities.

The wheel slip $\lambda$ and the speed $v$ are estimated online using an extended Kalman filter based on wheel speed and acceleration measurements. The central ECU monitors the commands given by the driver using the brake pedal. Essentially, it sets $T_{b}^{\max }=$ $k_{b} F_{b}^{*}$, where $F_{b}^{*}$ is the clamping force commanded by the driver using the brake pedal. The wheel slip controller is deactivated when the speed is below $1 \mathrm{~m} / \mathrm{s}$, and the controller state is reinitialized when the brake pedal is fully released.

\section{EXPERIMENTAL RESULTS AND REDESIGN}

In this section, we show test results for various road conditions. We only show results for a single front wheel, without any steering maneuvers but remark that similar or better performance is generally achieved at the rear wheels. We also propose some approaches to redesign and show experimental results that indicate the performance improvements that are achieved. 

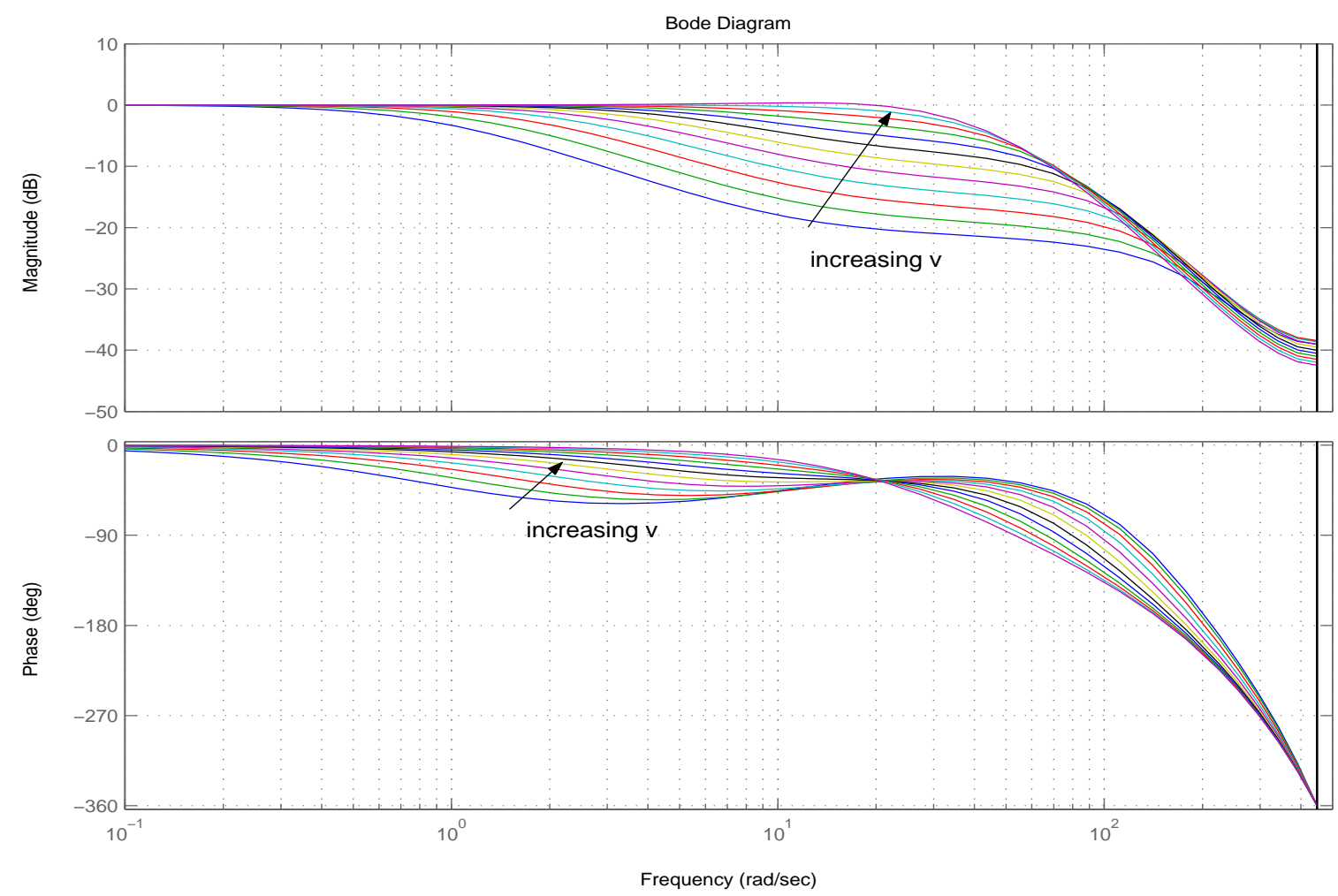

Fig. 9. Transfer function from reference to output, when the setpoint is somewhat to the left of the peak of the friction curve.

\section{A. Test results}

Figure 10 shows a test result with braking on dry asphalt. The slip setpoint is $\lambda^{*}=0.09$ and we note that the regulation is highly accurate and satisfactory. When the speed approaches zero, some variability in the slip emerges. Since the clamping force does not oscillate, we conclude that this is due to sensor noise that is known to increase as the speed goes to zero. However, the initial transient response is not satisfactory as the clamping force does not increase fast enough. Consequently, the slip is too low and the resulting friction force is too low in the interval $0.2 \leq t \leq 0.7$ leading to unnecessarily large braking distance. This is due to the significant model inaccuracy in the low-slip region due to the linearization, cf. Figure 2. A redesign of the slip controller is necessary for this region, and two approaches will be presented in Sections V-B and V-C.

Figure 11 shows a test result with braking on wet asphalt partly covered with a plastic coating (the setpoint is $\lambda^{*}=0.09$ ). We observe that the transient performance is much better than on dry asphalt, mainly because the steady-state value of the clamping force is closer to its initial state. We also observe that there is significantly more variability in the slip, although the slip is never larger than 0.4 (except when the velocity becomes very small). The variability is mainly due to an inhomogeneous road surface that provides external disturbances to the system. Overall, the performance is satisfactory.

Figures 12 and 13 show test results with braking on snow and ice respectively. The setpoint is $\lambda^{*}=0.07$ on snow and $\lambda^{*}=0.05$ on ice. Comparing the steady-state values for the clamping force $F_{b}$ we observe that on ice the friction coefficient is approximately $\mu_{H} \approx 0.1$, while on snow it is $\mu_{H} \approx 0.2$. At such low slip, the control system becomes very sensitive to disturbances such 
as an inhomogeneous road surface, sensor noise and actuator inaccuracies and nonlinearities due to internal friction and other phenomena. The transient performance is satisfactory in both cases and the regulation is still excellent.

\section{B. Controller initialization}

The first redesign idea is based on the observation that the initial state of the controller will play an important role in the initial transient. In the dry asphalt test in Figure 14, the slip setpoint is $\lambda^{*}=0.11$ and the initial state of the integrator $x_{1}(0)$ was set to a value that corresponds to the nominal steady-state clamping torque typically experienced at dry asphalt. We notice that the initial transient is significantly improved, but with an overshoot that might be eliminated by more accurate initialization. Similar ideas were exploited systematically in [14], [15], where a multiple model adaptive control approach with an estimator resetting rule was derived based on an adaptive control Lyapunov function. This allows automatic initialization of the controller state based on an estimate of the current road conditions, and it also provides automatic resetting when the friction coefficient changes abruptly during braking, for example on a dry surface with pathces of ice or water. So far, we have not developed a systematic initialization or resetting strategy, but we believe that this may be possible along the ideas in [14], [15].

\section{Off-equilibrium design}

The second redesign idea is based on the concept of off-equilibrium linearization and design in gain-scheduled control [21]. The idea is to introduce gain-scheduling that is particularly targeted at transient states in addition to conventional gain-scheduling that is targeted to near-equilibrium operation. In particular, we want to modify the controller during transients at low wheel slip values such that the setpoint is reached more rapidly. Thus, the controller switches to new gain matrices when the wheel slip $\lambda$ is lower than a given threshold, namely $0.6 \lambda^{*}$. These gain matrices are also designed using LQR based on local linearizations. However, the nominal $\hat{\lambda}$ is now on the steep part on the left side of the friction curve peak and, therefore, leads to a higher gain than near equilibria. Consequently, the transients are speeded up and the overall performance is improved as shown in the third dry asphalt test, Figure 15, where the wheel slip setpoint is $\lambda^{*}=0.11$. Again, there is some overshoot, but we believe this can be reduced by fine-tuning the switching thresholds and the off-equilibrium control design.

\section{CONCLUSIONS}

Using Lyapunov theory, frequency analysis and experimental verification, we have illustrated the performance and robustness of a gain-scheduled nonlinear wheel slip controller for automotive brakes. In order to achieve the robustness, the approach does not rely on strong knowledge of the tyre/road friction curve. Static uncertainty (due to unknown $\mu_{H}$ ) is eliminated using integral action, while dynamic uncertainty (due to the unknown shape of $\mu(\cdot)$ ) is handled by a robust design with sufficient stability margin by choosing the setpoint to the left of any friction curve peak.

Although a detailed comparison with existing off-the-shelf ABS has not been conducted, the present results are encouraging, in particular when taking into account the modest time taken to design, tune and commission this model-based approach. There is 
also room for significant improvements and extensions of the approach, both by fine tuning the controller and by adding a more sophisticated supervisory control level.

\section{ACKNOWLEDGEMENTS}

The work was sponsored by the European Commission under the ESPRIT LTR-project $28104 \mathrm{H}_{2} \mathrm{C}$.

\section{REFERENCES}

[1] M. Burckhardt, Fahrwerktechnik: Radschlupf-Regelsysteme, Vogel Verlag, Würzburg, 1993.

[2] SAE, “Antilock brake review," Tech. Rep. J2246, Society of Automotive Engineers, Warrendale PA, 1992.

[3] I. Petersen, T. A. Johansen, J. Kalkkuhl, and J. Lüdemann, "Wheel slip control in ABS brakes using gain scheduled constrained LQR," in European Control Conference, Porto, 2001.

[4] B. Hedenetz and R. Belschner, "Brake-by-wire without mechanical backup using a TTP communication network," in SAE International Congress and Exhibition, Detroit, 1998, p. 981109.

[5] R. Schwarz, Rekonstruktion der Bremskraft Bei Fahrzeugen mit Elektromechanisch Betätigten Radbremsen, Ph.D. thesis, Institut für Automatisierungstechnik der TU Darmstadt, 1999.

[6] R. Freeman, "Robust slip control for a single wheel," Tech. Rep. CCEC 95-0403, University of California, Santa Barbara, 1995.

[7] S. Drakunov, Ü. Özgüner, P. Dix, and B. Ashrafi, “ABS control using optimum search via sliding modes,” IEEE Trans. Control Systems Technology, vol. 3, no. 1, pp. 79-85, March 1995.

[8] C. C. de Wit, R. Horowitz, and P.Tsiotras, "Model-based observers for Tire/Road contact friction prediction," in In New Directions in Nonlinear Observer Design, H. Nijmeijer and T.I. Fossen, Eds., pp. 23-42. Springer Verlag, May 1999.

[9] Jingang Yi, Luis Alvarez, Roberto Horowitz, and Carlos Canudas de Wit, "Adaptive emergency braking control using a dynamical Tire/Road friction model," in IEEE Conference on Decision and Control, Sydney, 2000.

[10] Tor A. Johansen, Jens Kalkkuhl, Jens Lüdemann, and Idar Petersen, "Hybrid control strategies in ABS," in American Control Conference, Washington D.C., 2001.

[11] P. E. Wellstead and N. B. O. L. Pettit, “Analysis and redesign of an antilock brake system controller," IEE Proc. D, vol. 144 , pp. 413-426, 1997.

[12] M. C. Wu and M. C. Shih, "Hydraulic anti-lock braking control using the hybrid sliding-mode pulse width modulation pressure control method," Proc. Instn. Mech. Engrs., vol. 215, pp. 177-187, 2001.

[13] Hassan K. Khalil, Nonlinear Systems, Prentice Hall, 1996.

[14] J. Kalkkuhl, T. A. Johansen, J. Lüdemann, and A. Queda, "Nonlinear adaptive backstepping with estimator resetting using multiple observers," in Proc. Hybrid Systems, Computation and Control, Rome, 2001.

[15] J. Kalkkuhl, T. A. Johansen, and J. Lüdemann, "Improved transient performance of nonlinear adaptive backstepping using estimator resetting based on multiple models," IEEE Trans. Automatic Control, vol. 47, pp. 136-140, 2002.

[16] H. Kopetz and G. Grünsteidl, “TTP - a protocol for fault-tolerant real-time systems,” IEEE Computer, pp. 14-23, January 1994.

[17] I. Kaminer, A. M. Pascoal, P. P. Khargonekar, and E. E. Coleman, "A velocity algorithm for the implementation of gain-scheduled controllers," Automatica, vol. 31, pp. 1185-1191, 1995 .

[18] D. J. Leith and W. E. Leithead, "Appropriate realization of gain-scheduled controllers with application to wind turbine regulation," Int. J. Control, vol. 65, pp. 223-248, 1996.

[19] K. Ogata, Discrete-time control systems, Prentice Hall, 1995.

[20] T.A. Johansen, I. Petersen, and O. Slupphaug, "Explicit suboptimal linear quadratic regulation with state and input constraints," Automatica, vol. 38, 2002.

[21] T. A. Johansen, K. J. Hunt, P. J. Gawthrop, and H.Fritz, "Off-equilibrium linearisation and design of gain scheduled control with application to vehicle speed control," Control Engineering Practice, vol. 6, pp. 167-180, 1998. 


\section{APPENDIX}

In this appendix we prove that (47) implies $D(v)>0$ for all $v>0$.

$$
\begin{aligned}
& \dot{P}_{1,1}(v)=\frac{\partial P_{1,1}(v)}{\partial v} \dot{v}=\frac{\left(\alpha_{1}^{2}+\beta_{1}^{2} R^{-1}\left(Q_{2,2}(v)+\frac{2\left(Q_{1,1}(v) / R^{-1}\right)^{1 / 2}}{\beta_{1}} v\right)\right)^{1 / 2} \frac{d}{d v} Q_{1,1}(v)}{2 \beta_{1}\left(Q_{1,1}(v) R^{-1}\right)^{1 / 2}} \dot{v} \\
& +\frac{\left(Q_{1,1}(v) R^{-1}\right)^{1 / 2} \beta_{1}\left(\frac{d}{d v} Q_{2,2}(v)+\frac{2\left(Q_{1,1}(v) / R^{-1}\right)^{1 / 2}}{\beta_{1}}+\frac{\frac{d}{d v} Q_{1,1}(v)}{\beta_{1}\left(Q_{1,1}(v) R^{-1}\right)^{1 / 2}} v\right)}{2\left(\alpha_{1}^{2}+\beta_{1}^{2} R^{-1}\left(Q_{2,2}(v)+\frac{2\left(Q_{1,1}(v) / R^{-1}\right)^{1 / 2}}{\beta_{1}} v\right)\right)^{1 / 2}} \dot{v} \\
& \dot{P}_{1,2}(v)=\dot{P}_{2,1}(v)=\frac{\partial P_{1,2}(v)}{\partial v} \dot{v}=\left(\frac{\left(Q_{1,1}(v) / R^{-1}\right)^{1 / 2}}{\beta_{1}}+\frac{\frac{d}{d v} Q_{1,1}(v)}{2 \beta_{1}\left(Q_{1,1}(v) R^{-1}\right)^{1 / 2}} v\right) \dot{v} \\
& \dot{P}_{2,2}(v)=\frac{\alpha_{1}+\left(\alpha_{1}^{2}+\beta_{1}^{2} R^{-1}\left(Q_{2,2}(v)+\frac{2\left(Q_{1,1}(v) / R^{-1}\right)^{1 / 2}}{\beta_{1}} v\right)\right)^{1 / 2}}{\beta_{1}^{2} R^{-1}} \dot{v} \\
& +\frac{\left(\frac{d}{d v} Q_{2,2}(v)+\frac{2\left(Q_{1,1}(v) / R^{-1}\right)^{1 / 2}}{\beta_{1}}+\frac{\frac{d}{d v} Q_{1,1}(v)}{\beta_{1}\left(Q_{1,1}(v) R^{-1}\right)^{1 / 2}} v\right) v}{2\left(\alpha_{1}^{2}+\beta_{1}^{2} R^{-1}\left(Q_{2,2}(v)+\frac{2\left(Q_{1,1}(v) / R^{-1}\right)^{1 / 2}}{\beta_{1}} v\right)\right)^{1 / 2}} \dot{v}
\end{aligned}
$$

Now

$$
\begin{aligned}
& D(v)=\frac{\left(\alpha_{1}^{2}+\beta_{1}^{2} R^{-1}\left(Q_{2,2}(v)+\frac{2\left(Q_{1,1}(v) / R^{-1}\right)^{1 / 2}}{\beta_{1}} v\right)\right)^{1 / 2} \frac{d}{d v} Q_{1,1}(v) \alpha_{1}}{2 \beta_{1}\left(Q_{1,1}(v) R^{-1}\right)^{1 / 2} \beta_{1}^{2} R^{-1}} \\
& +\frac{\left(\alpha_{1}^{2}+\beta_{1}^{2} R^{-1}\left(Q_{2,2}(v)+\frac{2\left(Q_{1,1}(v) / R^{-1}\right)^{1 / 2}}{\beta_{1}} v\right)\right) \frac{d}{d v} Q_{1,1}(v)}{2 \beta_{1}\left(Q_{1,1}(v) R^{-1}\right)^{1 / 2} \beta_{1}^{2} R^{-1}} \\
& +\frac{\frac{d}{d v} Q_{1,1}(v)\left(\frac{d}{d v} Q_{2,2}(v)+\frac{2\left(Q_{1,1}(v) / R^{-1}\right)^{1 / 2}}{\beta_{1}}+\frac{\frac{d}{d v} Q_{1,1}(v)}{\beta_{1}\left(Q_{1,1}(v) R^{-1}\right)^{1 / 2}} v\right) v}{4 \beta_{1}\left(Q_{1,1}(v) R^{-1}\right)^{1 / 2}} \\
& +\frac{\left(Q_{1,1}(v) R^{-1}\right)^{1 / 2} \beta_{1}\left(\frac{d}{d v} Q_{2,2}(v)+\frac{2\left(Q_{1,1}(v) / R^{-1}\right)^{1 / 2}}{\beta_{1}}+\frac{\frac{d}{d v} Q_{1,1}(v)}{\beta_{1}\left(Q_{1,1}(v) R^{-1}\right)^{1 / 2}} v\right)}{2\left(\alpha_{1}^{2}+\beta_{1}^{2} R^{-1}\left(Q_{2,2}(v)+\frac{2\left(Q_{1,1}(v) / R^{-1}\right)^{1 / 2}}{\beta_{1}} v\right)\right)^{1 / 2}} \frac{\alpha_{1}}{\beta_{1}^{2} R^{-1}} \\
& +\frac{\left(Q_{1,1}(v) R^{-1}\right)^{1 / 2} \beta_{1}\left(\frac{d}{d v} Q_{2,2}(v)+\frac{2\left(Q_{1,1}(v) / R^{-1}\right)^{1 / 2}}{\beta_{1}}+\frac{\frac{d}{d v} Q_{1,1}(v)}{\beta_{1}\left(Q_{1,1}(v) R^{-1}\right)^{1 / 2}} v\right)}{2 \beta_{1}^{2} R^{-1}} \\
& +\frac{\left(Q_{1,1}(v) R^{-1}\right)^{1 / 2} \beta_{1}\left(\frac{d}{d v} Q_{2,2}(v)+\frac{2\left(Q_{1,1}(v) / R^{-1}\right)^{1 / 2}}{\beta_{1}}+\frac{\frac{d}{d v} Q_{1,1}(v)}{\beta_{1}\left(Q_{1,1}(v) R^{-1}\right)^{1 / 2}} v\right)^{2}}{4\left(\alpha_{1}^{2}+\beta_{1}^{2} R^{-1}\left(Q_{2,2}(v)+\frac{2\left(Q_{1,1}(v) / R^{-1}\right)^{1 / 2}}{\beta_{1}} v\right)\right)} v \\
& -\left(\frac{\left(Q_{1,1}(v) / R^{-1}\right)^{1 / 2}}{\beta_{1}}+\frac{\frac{d}{d v} Q_{1,1}(v)}{2 \beta_{1}\left(Q_{1,1}(v) R^{-1}\right)^{1 / 2}} v\right)^{2}
\end{aligned}
$$


Define the following positive variables:

$$
\begin{aligned}
A & =\alpha_{1}^{2}+D\left(Q_{2,2}(v)+\frac{2 C}{D} v\right)=\alpha_{1}^{2}+D Q_{2,2}(v)+2 C v \\
B & =\frac{d}{d v} Q_{2,2}(v)+\frac{2 C}{D}+\frac{\frac{d}{d v} Q_{1,1}(v)}{C} v \\
C & =\left(Q_{1,1}(v) R^{-1}\right)^{1 / 2} \beta_{1} \\
D & =\beta_{1}^{2} R^{-1}
\end{aligned}
$$

The above expression can then be rewritten to

$$
\begin{aligned}
D(v)= & \frac{A^{1 / 2} \frac{d}{d v} Q_{1,1}(v) \alpha_{1}}{2 C D}+\frac{A \frac{d}{d v} Q_{1,1}(v)}{2 C D}+\frac{\frac{d}{d v} Q_{1,1}(v) B v}{4 C} \\
& +\frac{\alpha_{1} B C}{2 A^{1 / 2} D}+\frac{C B}{2 D}+\frac{C B^{2}}{4 A} v-\left(\frac{C}{D}+\frac{\frac{d}{d v} Q_{1,1}(v)}{2 C} v\right)^{2}
\end{aligned}
$$

In (45) there are two possible negative factors involved: $\alpha_{1}$ and the last quadratic term with a negative $\operatorname{sign}\left(-P_{1,2}^{\prime}(v) P_{2,1}^{\prime}(v)\right)$. To cancel $P_{1,2}^{\prime}(v) P_{2,1}^{\prime}(v)$, parts from the 3rd and 5th term in (45) are used which gives

$$
\frac{\frac{d}{d v} Q_{1,1}(v) B v}{4 C}+\frac{C B}{2 D}-\left(\frac{C}{D}+\frac{\frac{d}{d v} Q_{1,1}(v)}{2 C} v\right)^{2}=\frac{\frac{d}{d v} Q_{2,2}(v) \frac{d}{d v} Q_{1,1}(v)}{4 C} v+\frac{d}{d v} Q_{2,2}(v)
$$

The following inequality thus ensures $D(v)>0$ :

$$
\begin{aligned}
D(v)= & \frac{A^{1 / 2} \frac{d}{d v} Q_{1,1}(v) \alpha_{1}}{2 C D}+\frac{A \frac{d}{d v} Q_{1,1}(v)}{2 C D}+\frac{\frac{d}{d v} Q_{2,2}(v) \frac{d}{d v} Q_{1,1}(v)}{4 C} v \\
& +\frac{\alpha_{1} B C}{2 A^{1 / 2} D}+\frac{d}{d v} Q_{2,2}(v)+\frac{C B^{2}}{4 A}>0
\end{aligned}
$$

where only $\alpha_{1}$ may have a negative value. 

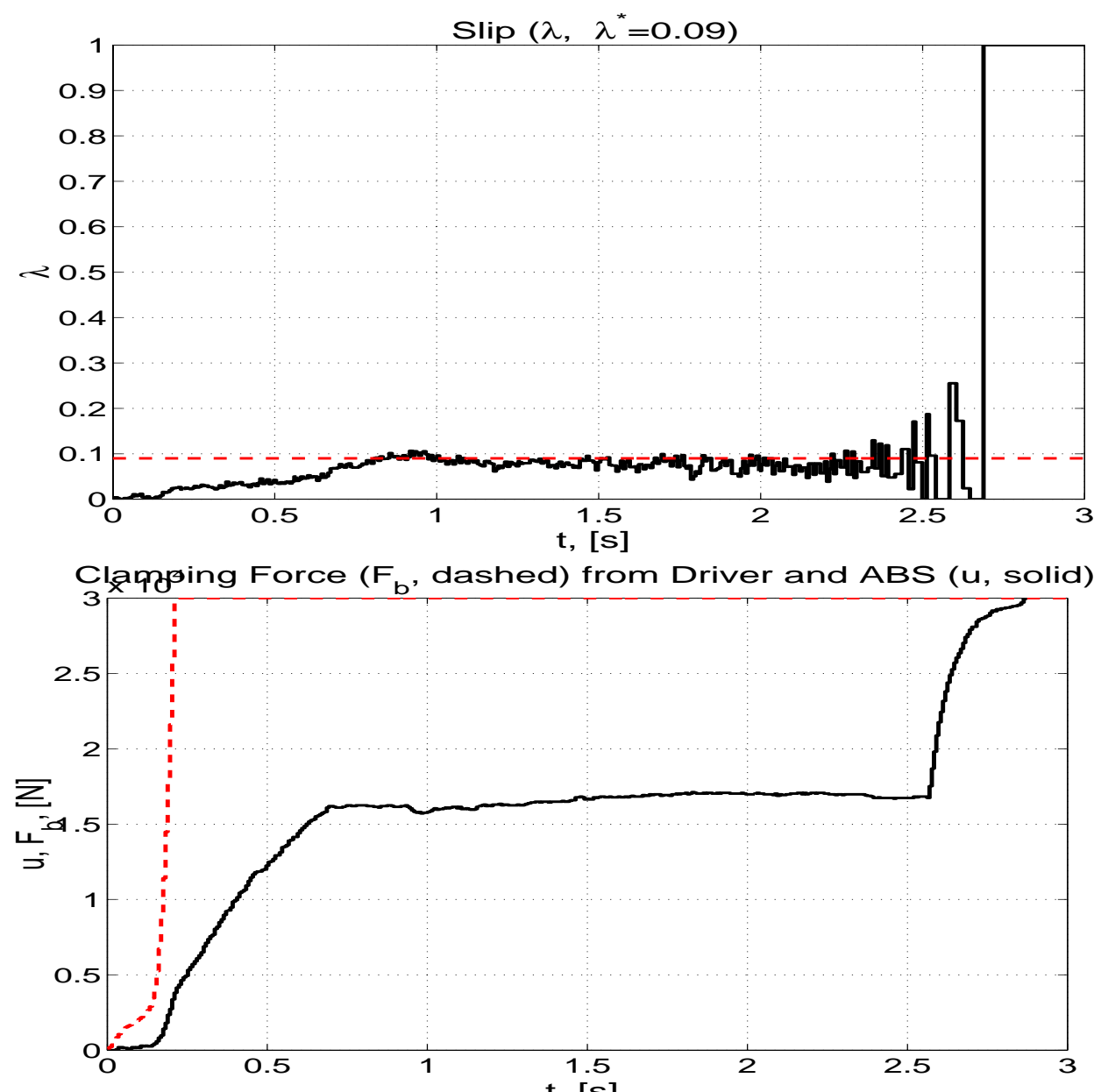

$\mathbf{t},[\mathbf{s}]$

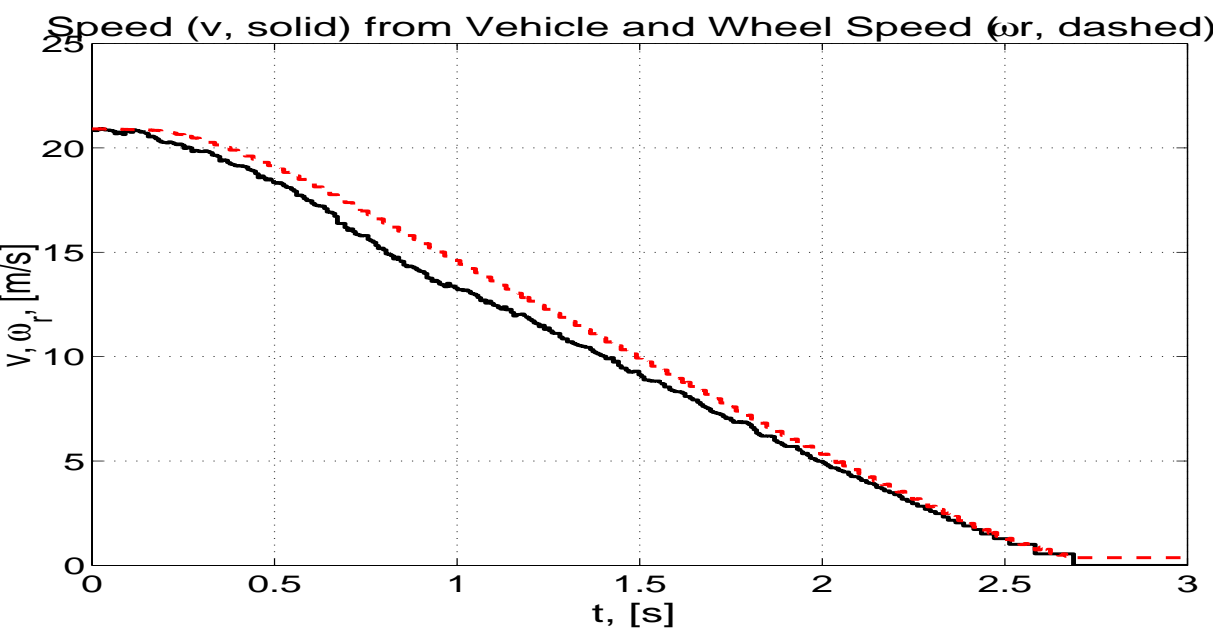

Fig. 10. Experimental results with braking on dry asphalt. 

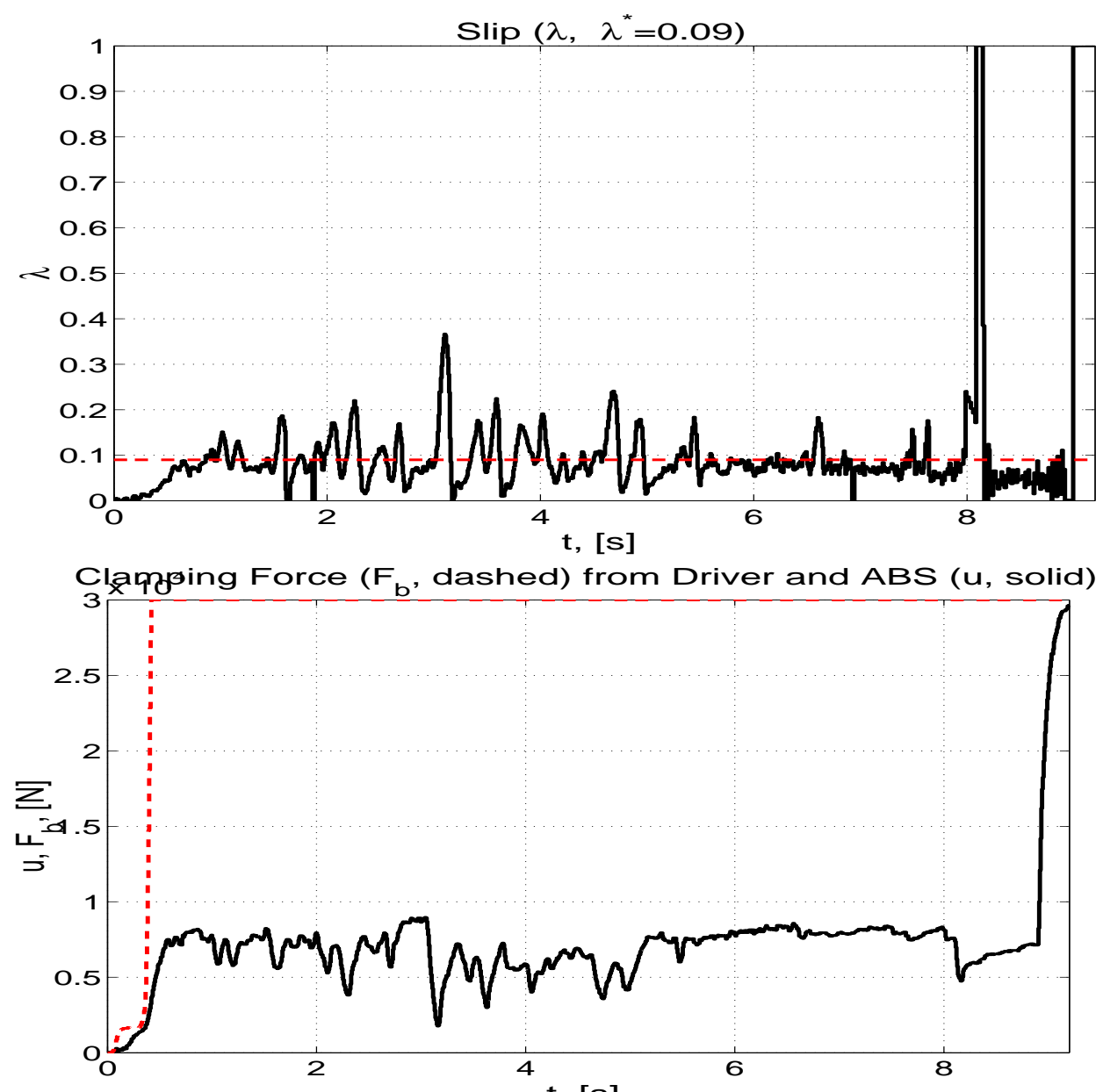

${ }_{25} 5$ eed ( $v$, solid) from Vehicle and Wheel Speed (or, dashed)

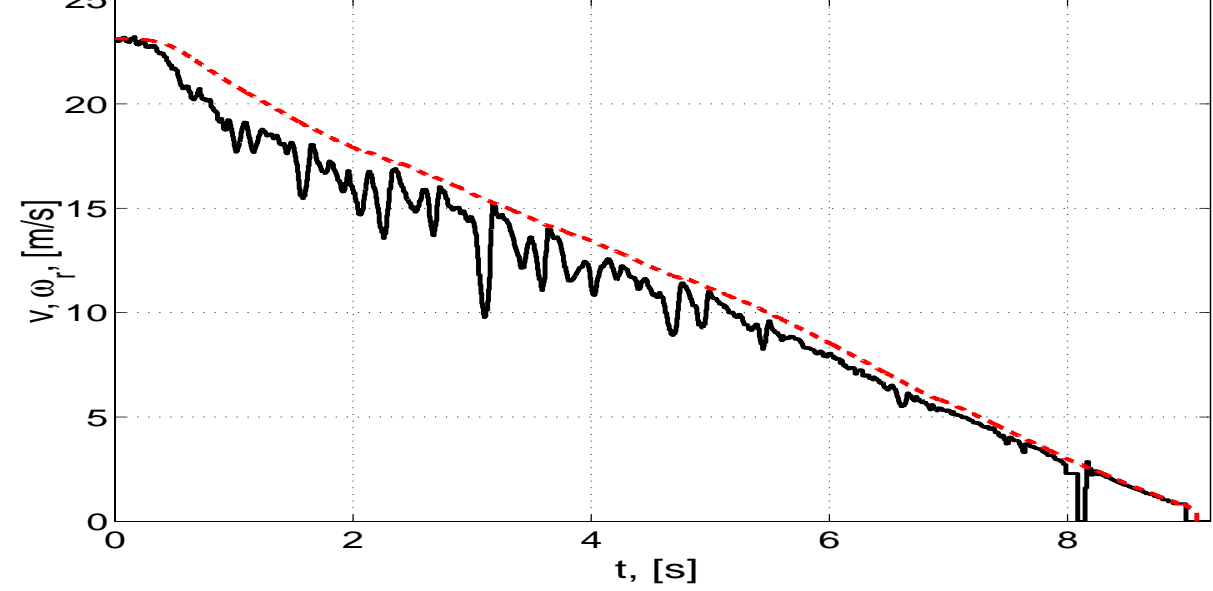

Fig. 11. Experimental results with braking on wet asphalt. 

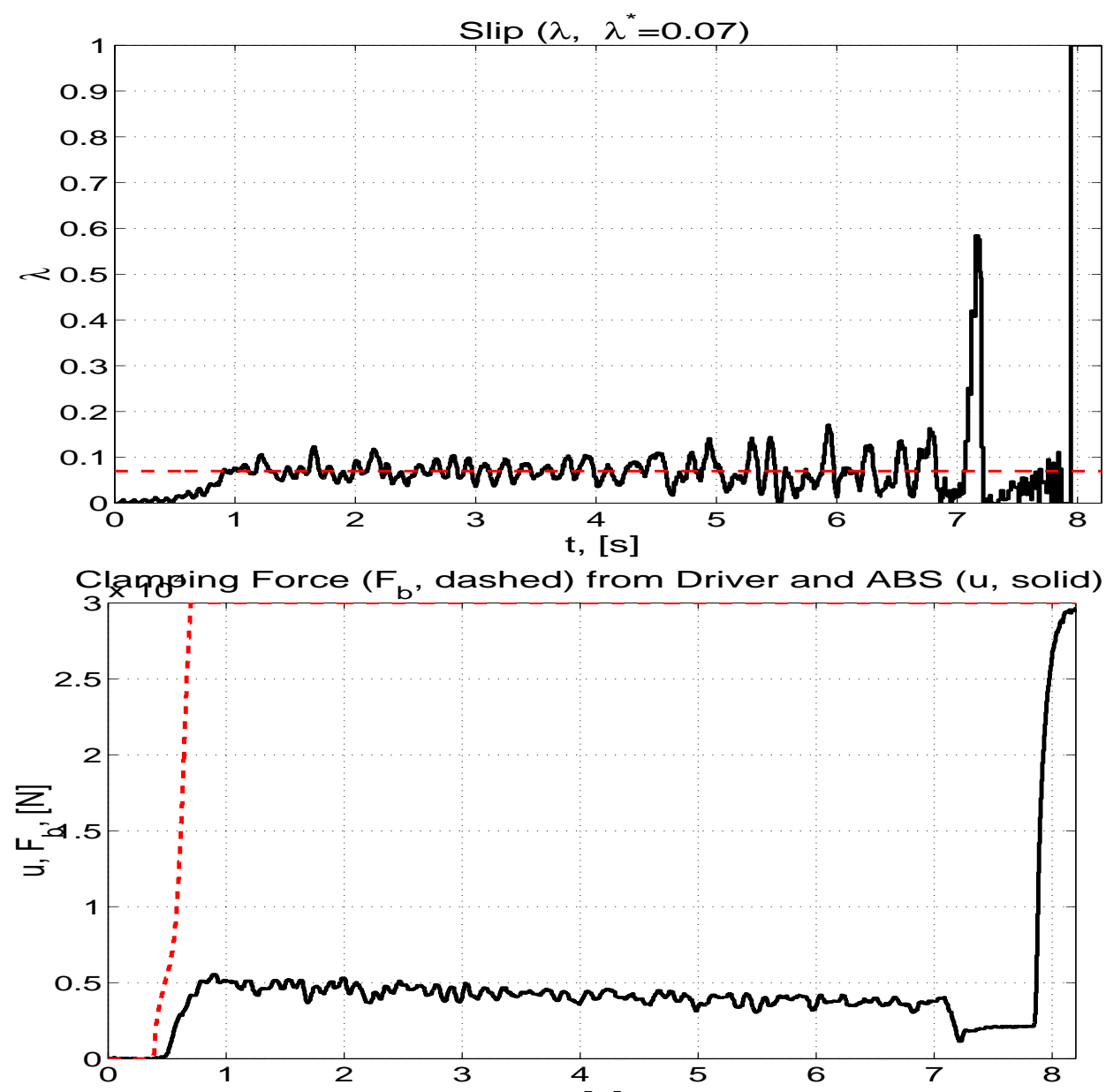

$t,[s]$

25 peed ( $v$, solid) from Vehicle and Wheel Speed (wr, dashed)

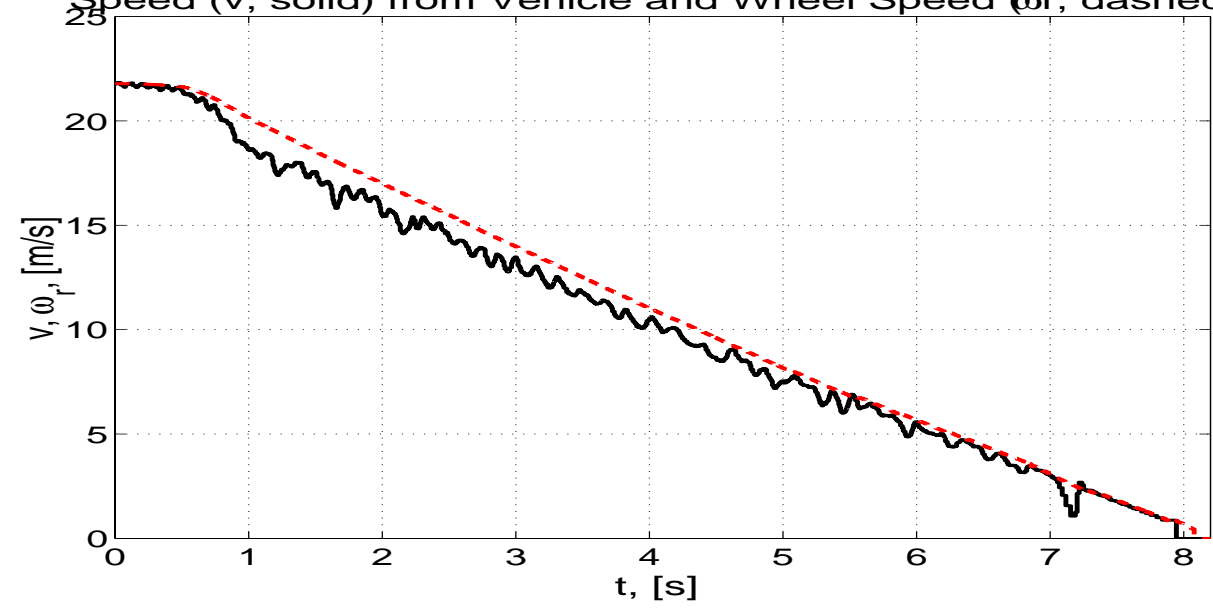

Fig. 12. Experimental results with braking on snow. 

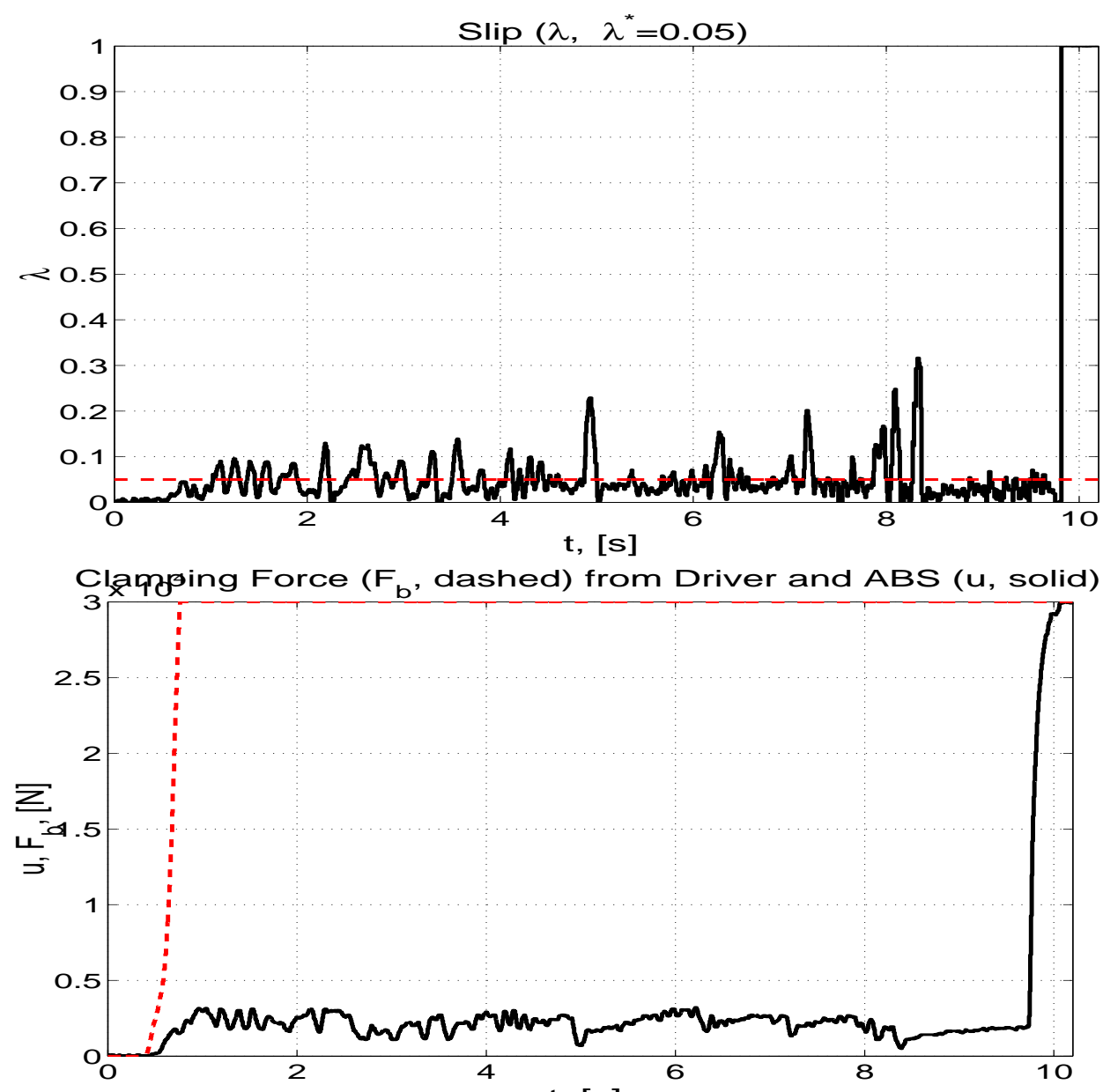

$t,[s]$

Speed ( $v$, solid) from Vehicle and Wheel Speed (wr, dashed)

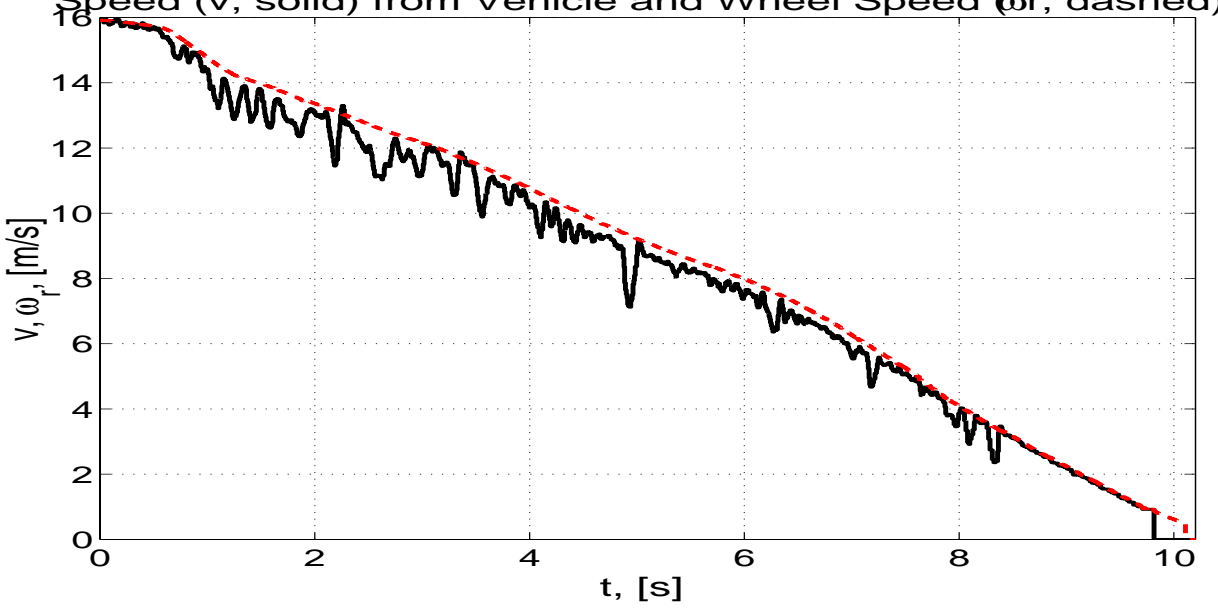

Fig. 13. Experimental results with braking on ice. 

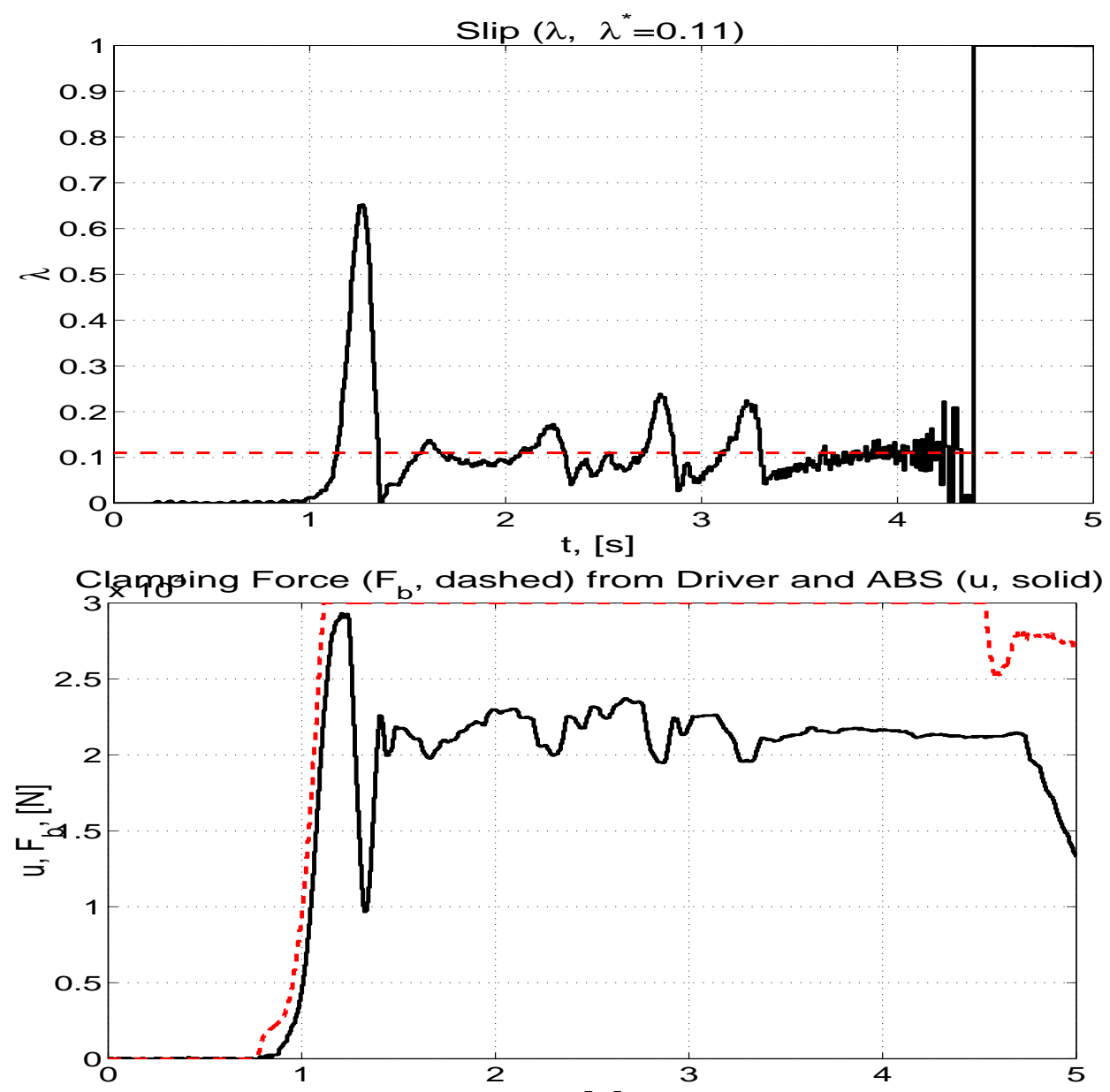

t, [s]

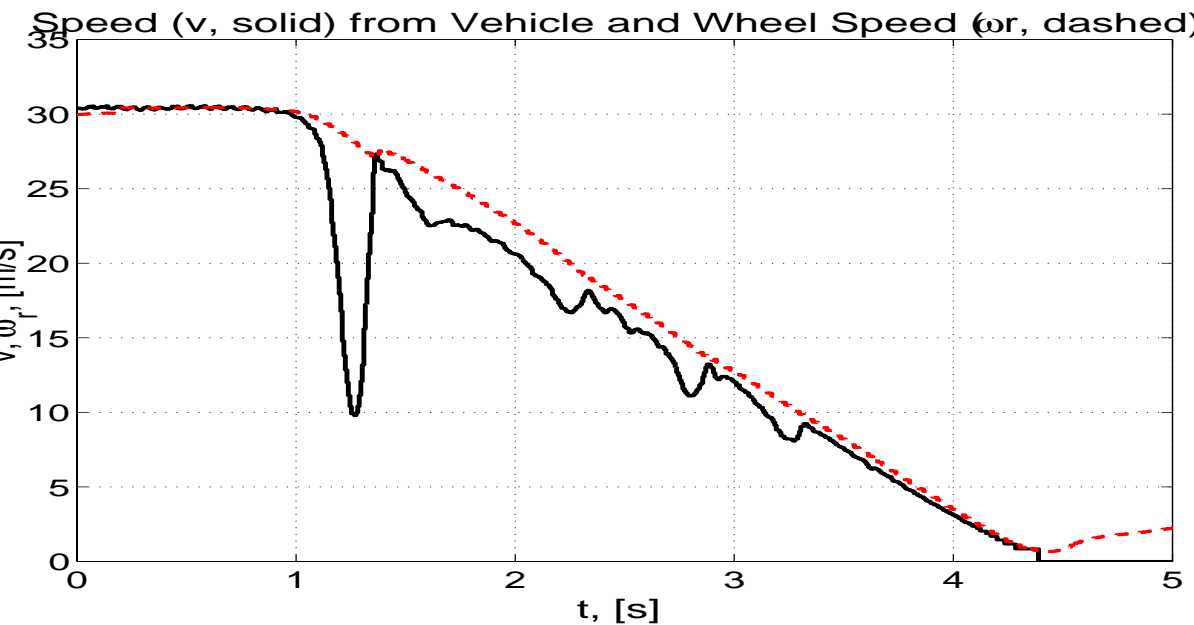

Fig. 14. Experimental results with braking on dry asphalt, with appropriate initialization of the slip error integrator. 

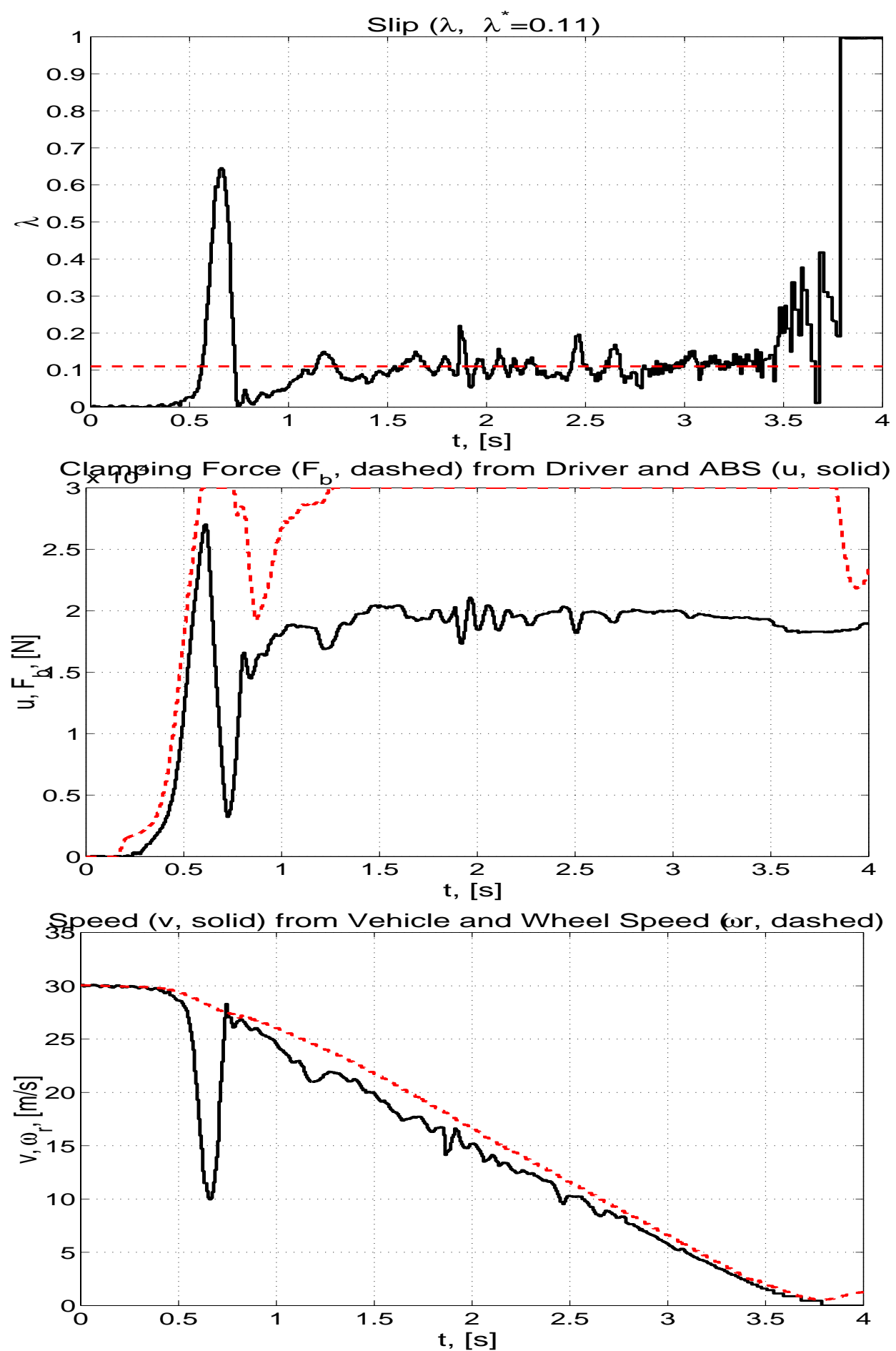

Fig. 15. Experimental results with braking on dry asphalt and scheduling on both slip and speed. 